\title{
Permutation Decoding and the Stopping Redundancy Hierarchy of Cyclic and Extended Cyclic Codes
}

\author{
Thorsten Hehn‡, Olgica Milenkovic $\dagger$, Stefan Laendner $\dagger$, and Johannes B. Huber $\neq$, \\ łUniversity of Erlangen-Nuremberg, Erlangen, Germany \\ $\dagger$ University of Colorado, Boulder, CO, USA
}

\begin{abstract}
We introduce the notion of the stopping redundancy hierarchy of a linear block code as a measure of the trade-off between performance and complexity of iterative decoding for the binary erasure channel. We derive lower and upper bounds for the stopping redundancy hierarchy via Lovász's Local Lemma and Bonferroni-type inequalities, and specialize them for codes with cyclic parity-check matrices. Based on the observed properties of parity-check matrices with good stopping redundancy characteristics, we develop a novel decoding technique, termed automorphism group decoding, that combines iterative message passing and permutation decoding. We also present bounds on the smallest number of permutations of an automorphism group decoder needed to correct any set of erasures up to a prescribed size. Simulation results demonstrate that for a large number of algebraic codes, the performance of the new decoding method is close to that of maximum likelihood decoding.
\end{abstract}

Index Terms: Automorphism Group, BCH Codes, Binary Erasure Channel, Cyclic Codes, Hamming Codes, Permutation Decoding, Stopping Redundancy Hierarchy, Stopping Sets.

\section{INTRODUCTION}

The error-correcting performance of linear block codes under iterative decoding depends jointly on a number of combinatorial properties of their parity-check matrices and corresponding Tanner graphs. These parameters include the minimum distance, weight distribution, girth, and diameter of the code graph. One exception is the binary erasure channel (BEC) transmission scenario, where only one class of combinatorial objects, termed stopping sets, completely characterizes the failure events during edge removal iterative decoding [1].

A stopping set is a collection of variable nodes in the Tanner graph of a code such that all check nodes in the subgraph induced by the variable nodes and their neighbors have degree at least two. The size of the smallest stopping set, as well as the distribution of stopping set sizes, depends on the particular form of the parity-check matrix used for decoding. Since including a large number of rows in the parity-check matrix of a code ensures increased flexibility in meeting predefined constraints on the structure of stopping sets, several authors recently proposed the use of redundant parity-check matrices for improving the performance of iterative decoders [2], [3], [4], [5], [6]. The effects of augmenting the sets of parity-checks in matrices from random ensembles were also studied in [7], [8].

Redundant rows in a parity-check matrix improve the performance of the decoder, but they also increase the overall time and hardware complexity of decoding. This motivates the study of achievable trade-offs between the number of redundant rows and the size of the smallest stopping set(s) in the chosen parity-check matrix. In this context, Schwartz and Vardy [2] introduced the notion of the stopping redundancy of a linear block code. The stopping redundancy represents the smallest number of codewords that span the dual code and constitute the rows of a matrix with no stopping sets of size smaller than the minimum distance of the code. The same authors also derived lower and upper bounds on the stopping redundancy, the latter growing exponentially with the co-dimension of the code for most examples considered. This finding raised the question if there exist codes for which one can significantly decrease the number of redundant rows in a parity-check matrix by slightly decreasing the size of its smallest stopping set.

Some results in this direction were independently derived by Weber and Abdel-Ghaffar [9], and Hollmann and Tolhuizen [10], who posed and partly solved the more complicated question of determining the smallest redundancy needed for decoding all correctable erasure patterns.

We extend the scope of the work in [10] by introducing the notion of the stopping redundancy hierarchy of a code. The stopping redundancy hierarchy characterizes the achievable trade-off between the stopping distance of a parity-check matrix and its number of rows. We focus our attention on codes with parity-check matrices of cyclic form. In particular, we analyze

Part of this work was presented at the 44th Annual Allerton Conference on Communication, Control, and Computing, Monticello, Illinois, Sept. 2006, and the IEEE International Symposium on Information Theory, Nice, France, June 2007. This work was supported in part by NSF Grant CCF-0514921 awarded to Olgica Milenkovic, by a research fellowship from the Institute for Information Transmission, University of Erlangen-Nuremberg, Erlangen, Germany, awarded to Stefan Laendner, and by a German Academic Exchange Service (DAAD) fellowship awarded to Thorsten Hehn. 
cyclic and extended cyclic codes, which have a rich mathematical structure and large minimum distance. We also improve a set of bounds presented in [10], pertaining to the family of $\mathrm{BCH}$ codes.

For cyclic codes, we derive the best known constructive upper bounds on the stopping redundancy hierarchy, using paritycheck matrices of cyclic form. Our construction methods imply that, rather than adding redundant rows to the parity-check matrix, one can change the belief propagation algorithm instead, by combining it with permutation decoding. Permutation decoding was first proposed for decoding of cyclic codes over the binary symmetric channel in [11]. In the new setting, permutation decoders can be seen as creating "virtual" redundant rows in the parity check-matrix of a code. The permutation algorithm itself operates on properly designed non-redundant parity-check matrices, and it has the property that it sequentially moves collections of erasures confined to stopping sets into positions that do not correspond to stopping sets.

The contributions of our work are three-fold. The first contribution consists in introducing the notion of the stopping redundancy hierarchy of a code and providing general upper and lower bounds on the elements of this ordered list. The second contribution lies in deriving upper and lower bounds on the stopping redundancy hierarchy of parity-check matrices of cyclic form. For the class of cyclic codes, we characterize the relationship between the stopping redundancy hierarchy and the minimum-weight codewords of their dual codes. As a third contribution, we demonstrate how the stopping redundancy hierarchy can be studied in the context of permutation decoding. More specifically, we describe a new decoding strategy, termed automorphism group decoding, which represents a combination of iterative message passing and permutation decoding. In connection with iterative permutation decoders, we study a new class of invariants of the automorphism group of a code, termed $s$-Stopping set Automorphism group Decoder (s-SAD) sets.

The algorithms described above are tested on the $[23,12,7]$ Golay code and the extended $[24,12,8]$ Golay code, a set of primitive $\mathrm{BCH}$ codes, as well as on a representative subclass of quadratic-residue codes [11]. Our findings indicate that automorphism group decoders exhibit near-maximum likelihood (ML) performance for short to moderate length cyclic codes.

The paper is organized as follows. Section $\amalg$ contains a summary of the terminology used throughout the paper and introduces the notion of the stopping redundancy hierarchy of a linear block code. A collection of general upper and lower bounds on the hierarchy is presented in Section III] Section IV outlines several methods for studying the stopping distance of cyclic codes. Section $\mathrm{V}$ introduces the automorphism redundancy, as well as the notion of s-PD and s-SAD sets. Automorphism group decoders are described in Section VI. Section VII provides a sampling of results regarding the performance of automorphism group decoders. Concluding remarks are given in Section VIII

\section{Definitions And Terminology}

We state next the definitions and terminology used throughout the paper. We restrict our attention to binary, linear blockcodes $\mathcal{C}$ with parameters $[n, k, d]$, used for signaling over the binary erasure channel (BEC). The erasure probability EP of a $\mathrm{BEC}$ is assumed to satisfy $0<\mathrm{EP}<1$. The iterative edge removal decoder operates on a suitably chosen parity-check matrix $\boldsymbol{H}$ of the code $\mathcal{C}$. For such a communication scenario, decoding errors are confined to subsets of codeword-coordinates termed stopping sets [1].

Stopping sets can be formally defined by referring either to the parity-check matrix of the code, or its underlying Tanner graph. For the former approach, we first introduce the notion of a restriction of a matrix.

Definition 2.1: Let the columns of $\boldsymbol{H}$ be indexed by $J=\{0, \ldots, n-1\}$. For a set $I \subseteq J,|I| \geq 1$, the restriction of $\boldsymbol{H}$ to $I$ is the matrix that consists of the set of columns of $\boldsymbol{H}$ indexed by the elements of $I$.

Definition 2.2: For a given parity-check matrix $\boldsymbol{H}$ of $\mathcal{C}$, a stopping set of size $\sigma$ is a subset $I(|I|=\sigma)$ of columns of $\boldsymbol{H}$, such that the restriction of $\boldsymbol{H}$ to $I$ avoids rows of Hamming weight one.

Alternatively, let $\boldsymbol{H}$ be a fixed parity-check matrix of $\mathcal{C}$, and let the bipartite graph $G=(L \cup R, E)$ be such that the columns of $\boldsymbol{H}$ are indexed by the variable nodes in $L$, and the rows of $\boldsymbol{H}$ are indexed by check nodes in $R$. For two vertices $i \in L$ and $j \in R,(i, j) \in E$ iff $H_{j, i}=1$. The graph $G$ constructed in this manner is called the Tanner graph of the code $\mathcal{C}$ with parity-check matrix $\boldsymbol{H}$. For $S \subset L$, we use $\Gamma(S)$ to denote the set of neighbors of $S$ in $R$.

Stopping sets can be defined using the notion of Tanner graphs as follows.

Definition 2.3: Given a bipartite graph $G=(L \cup R, E)$, we say that $S \subset L$ is a stopping set if the degree of each vertex in $\Gamma(S)$, restricted to the subgraph induced by $S \cup \Gamma(S)$, is greater than one.

The stopping distance of a parity-check matrix $\boldsymbol{H}$, and its underlying Tanner graph, is the cardinality of its smallest stopping set. Note that in the classical sense, the stopping distance does not actually represent a distance measure. Nevertheless, we adopt this terminology since it is by now standardly used in the coding theory literature.

The sizes and the number of stopping sets in a Tanner graph depends on the particular choice of the parity-check matrix. It is straightforward to see that adding rows to a fixed parity-check matrix $\boldsymbol{H}$ may only increase its stopping distance. To maintain the integrity of the code, the added rows must represent linear combinations of the base vectors in $\boldsymbol{H}$, and we refer to such rows as redundant rows (redundant parity-checks). A parity-check matrix $\boldsymbol{H}$ containing redundant parity-check equations is henceforth termed a redundant parity-check matrix. The phrase "parity-check matrix" is reserved for a matrix with the smallest possible number of rows, i.e., for a matrix of full row-rank; similarly, the symbol $m$ is reserved for the row-dimension of the (possibly) redundant parity-check matrix $\boldsymbol{H}$. Finally, we say that a matrix $\boldsymbol{H}$ (or, a subset of rows in $\boldsymbol{H}$ ) resolves a set of 
coordinates $I$ if the restriction of $\boldsymbol{H}$ (or the subset of rows of $\boldsymbol{H}$ ) to $I$ does not correspond to a stopping set. Observe that resolvability does not imply that all coordinates in $I$ can be retrieved if $I$ is not a stopping set. In the latter case, i.e., when $I$ is not a stopping set itself but "contains" one or more stopping sets, only a subset of its coordinates can be retrieved.

We are now ready to define the stopping redundancy hierarchy of a code.

Definition 2.4: Let $\mathcal{C}$ be a linear code with minimum distance $d$. For $\ell \leq d$, the $\ell$-th stopping redundancy of $\mathcal{C}$ is the smallest non-negative integer $\rho_{\ell}(\mathcal{C})$ such that there exists a (possibly redundant) parity-check matrix $\boldsymbol{H}$ of $\mathcal{C}$ with $\rho_{\ell}(\mathcal{C})$ rows and stopping distance at least $\ell$. The ordered set of integers

$$
\left(\rho_{1}(\mathcal{C}), \rho_{2}(\mathcal{C}), \rho_{3}(\mathcal{C}), \ldots, \rho_{d}(\mathcal{C})\right)
$$

is called the stopping redundancy hierarchy of $\mathcal{C}$. The order of an element in the stopping redundancy hierarchy is its position in the list. The integer $\rho_{d}(\mathcal{C})$ is denoted the stopping redundancy of $\mathcal{C}$, and was first introduced in [2].

For codes with minimum distance $d \geq 3$, no two columns of the parity-check matrix are identical nor is any of the columns equal to the all-zero vector. Therefore, $\rho_{1}(\mathcal{C})=\rho_{2}(\mathcal{C})=\rho_{3}(\mathcal{C})=n-k$. Consequently, only stopping redundancies of order larger than three are considered. Finding the stopping redundancy of a given order is very likely a complicated problem, since it was recently shown that computing the stopping distance of a matrix $\boldsymbol{H}$ is NP-hard and NP-hard to approximate [12], [13]. This is why we henceforth focus only on deriving upper and lower bounds on the elements of the hierarchy.

In the exposition to follow, we also frequently refer to the notion of (un)correctable erasure patterns [10], defined below. Only general properties of the code, but not the particular choices of their parity-check matrices, determine whether a pattern is correctable or not.

Definition 2.5: Let the entries of a codeword be indexed by $J=\{0, \ldots, n-1\}$, and define the support of the codeword as the set of its non-zero coordinates. A correctable erasure pattern is a set $I \subseteq J$ that does not properly contain the support of any codeword $c \in \mathcal{C}$. An uncorrectable erasure pattern is a set of positions that does not correspond to a correctable erasure pattern.

It is clear from the definition of uncorrectable erasure patterns that they represent erasure configurations that cannot be successfully reconstructed via ML decoding. An uncorrectable erasure pattern contains a stopping set, but the converse claim is not true.

\section{BOUNDS ON THE STOPPING REDUNDANCY HIERARCHY}

\section{A. Lower Bounds on the Stopping Redundancy Hierarchy}

We present next lower bounds on the $\ell$-th stopping redundancy, $\ell=4, \ldots, d$, of arbitrary binary linear codes. The first two bounds represent generalizations and extensions of lower bounds on the stopping redundancy derived in [2]. For completeness, we briefly state this result below.

Theorem 3.1: ([2]) Let $\mathcal{C}$ be a binary linear code with parameters $[n, k, d]$, and let

$$
\omega_{\sigma}=\max \left\{\lceil(n+1) / \sigma\rceil-1, d^{\perp}\right\}, \sigma=1, \ldots, d-1,
$$

where $d^{\perp}$ denotes the minimum distance of $\mathcal{C}^{\perp}$. Then

$$
\rho_{d}(\mathcal{C}) \geq \max \left(n-k, \max _{\sigma}\left(\frac{\left(\begin{array}{c}
n \\
\sigma
\end{array}\right)}{\omega_{\sigma}\left(\begin{array}{c}
n-\omega_{\sigma} \\
\sigma-1
\end{array}\right)}\right)\right), \sigma \in\{1, \ldots, d-1\} .
$$

It is instructive to briefly repeat the arguments leading to the results in Theorem 3.1 .

The bound is established by first noting that there exist $\left(\begin{array}{l}n \\ \sigma\end{array}\right)$ subsets of columns of $\boldsymbol{H}$ of cardinality $\sigma$. A row of Hamming weight $w$ can resolve exactly $w\left(\begin{array}{c}n-w \\ \sigma-1\end{array}\right)$ stopping sets of size $\sigma$. A simple application of the union bound shows that

$$
\left(\begin{array}{l}
n \\
\sigma
\end{array}\right) \leq \sum_{i=1}^{\rho_{d}(\mathcal{C})} w(i)\left(\begin{array}{c}
n-w(i) \\
\sigma-1
\end{array}\right) \leq \rho_{d}(\mathcal{C}) \max _{i}\left(w(i)\left(\begin{array}{c}
n-w(i) \\
\sigma-1
\end{array}\right)\right),
$$

where $w(i)$ denotes the weight of the $i$-th row in $\boldsymbol{H}$. The result follows by noting that this expression has to hold for all $\sigma \leq d-1$ and that the upper bound is maximized by choosing the row weights $w(i)$ according to Equation (1).

Theorem 3.2: Let $\mathcal{C}$ be a binary $[n, k, d]$ linear code, and let

$$
\omega_{\sigma}=\max \left\{\lceil(n+1) / \sigma\rceil-1, d^{\perp}\right\}, \sigma=1, \ldots, d-1,
$$

where $d^{\perp}$ denotes the minimum distance of $\mathcal{C}^{\perp}$. Then

$$
\rho_{\ell}(\mathcal{C}) \geq \max \left(n-k, \max _{\sigma}\left(\frac{\left(\begin{array}{c}
n \\
\sigma
\end{array}\right)}{\omega_{\sigma}\left(\begin{array}{c}
n-\omega_{\sigma} \\
\sigma-1
\end{array}\right)}\right)\right), \sigma \in\{1, \ldots, \ell-1\} .
$$

Note that Theorem 3.2 is a straightforward generalization of Theorem 3.1 Hence, the proof of this result follows the proof of Theorem 3.1 very closely and is therefore omitted. Both Theorem 3.1 and 3.2 can be shown to be very loose through numerous examples. 
Tighter lower bounds on the stopping redundancy hierarchy can be derived by means of a more precise count of the number of subsets of columns of $\boldsymbol{H}$ that do not correspond to stopping sets. More specifically, the number of such subsets equals the cardinality of the union of all subsets for which the restriction of one individual row of $\boldsymbol{H}$ does not have weight one. The result of Theorem 3.2 represents a weak bound on the stopping redundancy hierarchy due to the use of the inherently loose union bound. More accurate bounds can be calculated by invoking the principle of inclusion-exclusion, stated below.

Theorem 3.3 ([14]): Let $|A|$ denote the cardinality of the set $A$, and let $V$ be the set $\{1, \ldots,|V|\}$. For a family of sets $\left\{A_{v}\right\}_{v \in V}$, the principle of inclusion-exclusion (PIE) asserts that

$$
\left|\bigcup_{v \in V} A_{v}\right|=\sum_{\substack{I \subseteq V \\ I \neq \emptyset}}(-1)^{|I|-1}\left|\bigcap_{v \in I} A_{v}\right| \text {. }
$$

Let $\Sigma_{\sigma, i}$ denote the set of stopping sets of size $\sigma$ resolved by the $i$-th row of $\boldsymbol{H}$. Also, let $R=\{1,2, \ldots, m\}$ denote the set of row indices of $\boldsymbol{H}$, and let $D_{j}$ denote the set of all $j$-subsets of $R$.

The number of stopping sets of size $\sigma$ resolved by $\boldsymbol{H}$ can be found from the PIE equation as

$$
\left|\bigcup_{i=1}^{m} \Sigma_{\sigma, i}\right|=\sum_{\substack{I \subseteq R \\ I \neq \emptyset}}(-1)^{|I|-1}\left|\cap_{i \in I} \Sigma_{\sigma, i}\right|=\sum_{j=1}^{m}(-1)^{j-1} S_{\sigma, j}, \quad \text { where } \quad S_{\sigma, j}=\sum_{T \in D_{j}} s_{\sigma, T} .
$$

Here, $s_{\sigma, T}$ denotes the number of stopping sets of size $\sigma$ resolved by the rows indexed by elements of the set $T$.

There exists a simple relationship between the number of stopping sets resolved by a given parity-check matrix and the $\ell$-th stopping redundancy hierarchy of the underlying code. The latter equals the smallest possible dimension $m$ of a matrix that resolves all stopping sets of size $\sigma$ smaller than $\ell$, i.e. an $m$ such that

$$
\left|\bigcup_{i=1}^{m} \Sigma_{\sigma, i}\right|=\left(\begin{array}{l}
n \\
\sigma
\end{array}\right), \quad 1 \leq \sigma<\ell .
$$

The exact calculation of the number of stopping sets resolved by an arbitrary collection of $j$ rows is very complex, unless the code has certain regularity properties. One such property is that all collections of $j$ codewords from $\mathcal{C}^{\perp}$ resolve the same number of stopping sets. In this case, $S_{\sigma, j}=\left(\begin{array}{c}m \\ j\end{array}\right) \cdot s_{\sigma}$, where $s_{\sigma}$ denotes the number of stopping sets of size $\sigma$ resolved by the rows indexed with $1, \ldots, j$.

The PIE is frequently applied in the form of an upper or lower bound: this is accomplished by neglecting terms involving intersections of sets including more than $b$ terms, i.e. by retaining only those terms for which $|I| \leq b[14]$. Whenever the sign of the first omitted term is negative, the resulting expression represents an upper bound on the left hand side of the PIE formula. Otherwise, the obtained formula represents a lower bound. Inequalities obtained in this manner are known as Bonferroni inequalities.

There exists a rich body of work on Bonferroni inequalities [14], [15], and one of its forms most amenable for stopping set analysis is given below.

$$
\left|\bigcup_{v \in V} A_{v}\right| \leq \sum_{\substack{I \subseteq V \\|I|=1}}(-1)^{|I|-1}\left|\bigcap_{v \in I} A_{v}\right|+\frac{2}{|V|} \cdot \sum_{\substack{I \subseteq V \\|I|=2}}(-1)^{|I|-1}\left|\bigcap_{v \in I} A_{v}\right| .
$$

This allows us to state the following result.

Proposition 3.4: The number of distinct stopping sets of size $\sigma$ resolved by a parity-check matrix $\boldsymbol{H}$ with $m$ rows satisfies

$$
\left|\bigcup_{i=1}^{m} \Sigma_{\sigma, i}\right| \leq \sum_{i=1}^{m}\left|\Sigma_{\sigma, i}\right|-\frac{2}{m} \cdot \sum_{\substack{i, j=1 \\ i<j}}^{m}\left|\Sigma_{\sigma, i} \cap \Sigma_{\sigma, j}\right| .
$$

The $\ell$-th order stopping redundancy $\rho_{\ell}(\mathcal{C})$ is lower bounded by the smallest integer $m$ for which the right hand side of Equation (3) equals or exceeds $\left(\begin{array}{l}n \\ \sigma\end{array}\right)$, for all $\sigma<\ell$.

Proof: Equation (3) is a straightforward consequence of Equation (2); the second observation follows from the fact that, in order for a matrix with $m$ rows to have stopping distance at least $\sigma$, the upper bound on the number of stopping sets it resolves must exceed the total number of stopping sets of size $\sigma$ or less.

The above results will be specialized for codes with cyclic parity-check matrices in Section IV General expressions for the set intersection cardinalities of (3) are presented below. They reveal that the weights of the rows, as well as the gaps (spacings) between non-zero elements in the rows of a (redundant) parity-check matrix $\boldsymbol{H}$ bear a strong influence on its stopping distance hierarchy. 
Definition 3.5: Let $O_{l}$ and $Z_{l}, l \in\{1, \ldots, m\}$, denote the sets of positions of ones and zeros in the $l$-th row of the paritycheck matrix $\boldsymbol{H}$ with $m$ rows, respectively. The intersection number $\left|X_{i} \cap Y_{j}\right|$ of $\boldsymbol{H}, X, Y \in\{O, Z\}, i, j \in\{1, \ldots, m\}$, is defined as the cardinality of the set $P \subseteq J$ of positions $p$ such that $H_{i, p}=X$ and $H_{j, p}=Y$ for all $p \in P$.

Lemma 3.6: The number of stopping sets of size $\sigma$, resolved by the $i$-th row of $\boldsymbol{H}$ of weight $\omega(i)$, equals

$$
\left|\Sigma_{\sigma, i}\right|=\omega(i) \cdot\left(\begin{array}{c}
n-\omega(i) \\
\sigma-1
\end{array}\right) .
$$

The number of stopping sets of size $\sigma$, resolved jointly by two rows of $\boldsymbol{H}$ indexed by $i$ and $j$, equals

$$
\left|\Sigma_{\sigma, i} \cap \Sigma_{\sigma, j}\right|=\left|O_{i} \cap O_{j}\right| \cdot\left(\begin{array}{c}
\left|Z_{i} \cap Z_{j}\right| \\
\sigma-1
\end{array}\right)+\left|O_{i} \cap Z_{j}\right| \cdot\left|Z_{i} \cap O_{j}\right| \cdot\left(\begin{array}{c}
\left|Z_{i} \cap Z_{j}\right| \\
\sigma-2
\end{array}\right) .
$$

Proof: The first part follows from the fact that a stopping set of size $\sigma$ can be resolved by the restriction of a row of weight one, with the one-entry chosen among $\omega(i)$ options and the remaining zeros from $\left(\begin{array}{c}n-\omega(i) \\ \sigma-1\end{array}\right)$ choices. The second claim is a consequence of the following observation: a pair of rows resolves the same stopping set if either both rows share a " 1 " and all the " 0 "s in the remaining positions of the stopping set; or, if within the support of the stopping set the two rows share all except for two positions for the " 0 " symbols, and have two non-overlapping positions with the symbol " 1 ".

The expressions in Lemma (3.6) can be further simplified by assuming that all rows in $\boldsymbol{H}$ have weight $\omega$ (provided that $\mathcal{C}^{\perp}$ has sufficiently many codewords of weight $\omega$ ), and by writing

$$
\left|\Sigma_{P}\right|=\max _{\{i, j\}}\left|\Sigma_{\sigma, i} \cap \Sigma_{\sigma, j}\right| .
$$

Under these assumptions,

$$
\left|\bigcup_{i=1}^{m} \Sigma_{\sigma, i}\right| \leq m \cdot \omega \cdot\left(\begin{array}{c}
n-\omega \\
\sigma-1
\end{array}\right)-\frac{2}{m} \cdot\left(\begin{array}{c}
m \\
2
\end{array}\right) \cdot\left|\Sigma_{P}\right|,
$$

where $\left|\Sigma_{P}\right|$ can be found based on the formula in Lemma 3.6

A lower bound on the $\ell$-th stopping redundancy can be obtained from Lemma 3.6 by observing that the upper bound in (4) has to exceed $\left(\begin{array}{l}n \\ \sigma\end{array}\right)$ for all $\sigma \leq \ell$. As a consequence,

$$
\left.\rho_{\ell}(\mathcal{C})\right|_{\omega(i)=\omega} \geq \max _{\sigma<\ell}\left\lceil\frac{\left(\begin{array}{l}
n \\
\sigma
\end{array}\right)-\left|\Sigma_{P}\right|}{\omega\left(\begin{array}{l}
n-\omega \\
\sigma-1
\end{array}\right)-\left|\Sigma_{P}\right|}\right\rceil,
$$

where the qualifier $\omega(i)=\omega$ denotes that a constant codeword weight $\omega$ was assumed for this bound. The previous analysis shows that, in order to resolve a large number of stopping sets by a (redundant) parity-check matrix $\boldsymbol{H}$ with a small number of rows, one has to minimize the number of stopping sets resolved jointly by subsets of rows. Therefore, small sizes for the intersections of the supports of pairs, triples, etc., of rows are desirable. This is why we henceforth restrict our attention to matrices $\boldsymbol{H}$ that contain minimum weight codewords of $\mathcal{C}^{\perp}$ as their rows.

It is also worth pointing out that for many codes the codewords of minimum weight or other fixed weight represent a design [11]. In those cases, the intersection numbers of the minimum weight codewords can be obtained from the parameters of the design. Examples supporting this observation, pertaining to Quadratic Residue (QR) codes and the Golay codes [11], are given in subsequent sections.

Example 3.1: Consider a parity-check matrix of the form shown below:

$$
\boldsymbol{H}=\left(\begin{array}{ccccc}
1 & 0 & 0 & 0 & 1 \\
1 & 0 & 0 & 1 & 0
\end{array}\right)
$$

The columns indexed by $\{1,2,3\}$ have a unique row-restriction, namely "100". The columns indexed by $\{2,4,5\}$ and $\{3,4,5\}$ have row restrictions " 001 " and "010", respectively. Consequently, both rows simultaneously resolve stopping sets indexed by the described set of columns. More precisely, both rows resolve the three restrictions indexed by $\{1,2,3\},\{2,4,5\}$, and $\{3,4,5\}$, while all other restrictions are resolved by at most one of the rows.

Example 3.2: Consider the family of $\left[2^{s}-1, s, 2^{s-1}\right]$ simplex codes. Since simplex codes have constant codeword weight $2^{s-1}$, for all distinct indices $i$ and $j$, the intersection numbers are given as $\left|O_{i} \cap O_{j}\right|=2^{s-2},\left|O_{i} \cap Z_{j}\right|=\left|Z_{i} \cap O_{j}\right|=2^{s-2}$, and $\left|Z_{i} \cap Z_{j}\right|=2^{s}-1-3 \cdot 2^{s-2}=2^{s-2}-1$. The bound in (5) shows that for $i \neq j$, the dual code of a simplex code, a $\left[2^{s}-1,2^{s}-s-1,3\right]$ Hamming code $\mathcal{C}(s)$, satisfies

$$
\left|\Sigma_{\sigma, i} \cap \Sigma_{\sigma, j}\right|=\left|\Sigma_{\sigma, 1} \cap \Sigma_{\sigma, 2}\right|=2^{s-2}\left[\left(\begin{array}{c}
2^{s-2}-1 \\
\sigma-1
\end{array}\right)+2^{s-2}\left(\begin{array}{c}
2^{s-2}-1 \\
\sigma-2
\end{array}\right)\right],
$$


and that, consequently,

$$
\left.\rho_{3}(\mathcal{C}(s))\right|_{\omega_{i}=2^{s-1}} \geq \max _{\sigma<3}\left[\frac{\left(\begin{array}{c}
2^{s}-1 \\
\sigma
\end{array}\right)-2^{s-2} \cdot\left[\left(\begin{array}{c}
2^{s-2}-1 \\
\sigma-1
\end{array}\right)+2^{s-2} \cdot\left(\begin{array}{c}
2^{s-2}-1 \\
\sigma-2
\end{array}\right)\right]}{2^{s-1} \cdot\left(\begin{array}{c}
2^{s-1}-1 \\
\sigma-1
\end{array}\right)-2^{s-2} \cdot\left[\left(\begin{array}{c}
2^{s-2}-1 \\
\sigma-1
\end{array}\right)+2^{s-2} \cdot\left(\begin{array}{c}
2^{s-2}-1 \\
\sigma-2
\end{array}\right)\right]}\right] .
$$

Simple evaluation of the bound reveals that

$$
\left.\rho_{3}(\mathcal{C}(s))\right|_{\omega_{i}=2^{s-1}} \geq \max \left(\left\lceil 3-2^{2-s}\right\rceil,\left\lceil\frac{3-5 \cdot 2^{1-s}}{1-2^{1-s}}\right\rceil\right)
$$

which implies the trivial result $\rho_{3}(\mathcal{C}(s)) \geq 3, s>1$. This illustrates the fact that PIE-based lower bounds can be arbitrarily weak, since it is known that $\rho_{3}(\mathcal{C}(s))=s$.

\section{B. Upper Bounds on the Stopping Redundancy Hierarchy}

Upper bounds on the stopping redundancy hierarchy can be derived by invoking probabilistic methods [16]. Our derivations follow the framework we developed in [7], based on Lovász Local Lemma [17], [16]. Related probabilistic techniques were also used for deriving upper bounds on the stopping redundancy in [4].

For subsequent derivations, we need the following results, known as Lovász Local Lemma (LLL), as well as the highprobability variation of LLL.

Lemma 3.7: Let $E_{1}, E_{2}, \ldots, E_{N}$ be a set of events in an arbitrary probability space. Suppose that each event $E_{i}$ is independent of all other events $E_{j}$, except for at most $\tau$ of them, and that

$$
P\left\{E_{i}\right\} \leq p, \quad \forall 1 \leq i \leq N .
$$

If

$$
\text { e } p(\tau+1) \leq 1,
$$

where e is the base of the natural logarithm, then $P\left\{\bigcap_{i=1}^{N} \bar{E}_{i}\right\}>0$.

Furthermore, let $0<\epsilon<1$. If

$$
P\left\{E_{i}\right\} \leq \frac{\epsilon}{N}\left(1-\frac{\epsilon}{N}\right)^{\tau}, 1 \leq i \leq N,
$$

then $P\left\{\bigcap_{i=1}^{N} \bar{E}_{i}\right\}>1-\epsilon$.

Based on Lovász Local Lemma (6) and its high-probability variation (8), one can obtain the following bounds on the $\ell$-th stopping redundancy of a code, for $\ell \leq\left\lfloor\frac{d+1}{2}\right\rfloor$. The derivations are straightforward, and based on associating stopping distance properties of restrictions of a parity-check matrix with the events $E_{i}$ described in the statement of LLL.

Theorem 3.8: Let $\mathcal{C}$ be an $[n, k, d]$ code. If $\ell \leq\left\lfloor\frac{d+1}{2}\right\rfloor$ and

$$
m \geq \frac{1+\log \sum_{j=1}^{\ell-1}\left(\left(\begin{array}{c}
n \\
j
\end{array}\right)-\left(\begin{array}{c}
n-j \\
j
\end{array}\right)\right)}{-\log \left(1-\frac{\ell-1}{2^{\ell-1}}\right)}+n-k-\ell+1,
$$

then $\rho_{\ell}(\mathcal{C}) \leq m$. Alternatively, if the conditions of the theorem are fulfilled, then there exists at least one parity-check matrix with $m$ rows and stopping distance at least $\ell$.

The proof of this non-constructive bound is given in Appendix [. An asymptotic estimate of the above bound is presented in Appendix $\Pi$ for codes with $d=$ const. $\cdot n$.

One way to make the finding of Theorem 3.8 more useful for practical purposes is to generalize it by invoking the high probability version of LLL, as stated below.

Theorem 3.9: Let $\mathcal{C}$ be an $[n, k, d]$ code, let $\ell \leq\left\lfloor\frac{d+1}{2}\right\rfloor$, and assume that $m$ fulfills the condition

$$
m \geq \frac{\left.\log \frac{\epsilon}{\sum_{j=1}^{\ell-1}\left(\begin{array}{l}
n \\
j
\end{array}\right)}+\left(\begin{array}{c}
\ell-1 \\
j=1
\end{array}\left[\begin{array}{c}
n \\
j
\end{array}\right)-\left(\begin{array}{c}
n-j \\
j
\end{array}\right)-1\right]\right) \cdot \log \left(1-\frac{\epsilon}{\sum_{j=1}^{\ell-1}\left(\begin{array}{c}
n \\
j
\end{array}\right)}\right)}{\log \left(1-\frac{\ell-1}{2^{\ell-1}}\right)} .
$$

Then the probability that a parity-check matrix consisting of $m$ randomly chosen codewords of the dual code (with possible repetitions of a codeword) has stopping distance $\ell$ is at least $1-\epsilon$. With at most $n-k-\ell+1$ additional rows, the matrix also has rank $n-k$ and represents a valid parity-check matrix of the code $\mathcal{C}$.

The proof of the theorem is given in Appendix [III Numerical results for a selected set of values of $\ell$ is given in Section IV

As the results of Theorems 3.8 and 3.9 are non-constructive and conditioned on $\ell \leq\left\lfloor\frac{d+1}{2}\right\rfloor$, we present another method for finding upper bounds on the $\ell$-th stopping redundancy. This second class of upper bounds on the stopping redundancy hierarchy of a code is constructive in nature, and based on the following result. 
Theorem 3.10: ([2]) Let $\mathcal{C}$ be a binary linear code with parameters $[n, k, d]$, with $d>3$. Then

$$
\rho_{d}(\mathcal{C}) \leq\left(\begin{array}{c}
n-k \\
1
\end{array}\right)+\ldots+\left(\begin{array}{l}
n-k \\
d-2
\end{array}\right)
$$

The proof of the bound in Theorem 3.10 is constructive: one starts with an arbitrary parity-check matrix $\boldsymbol{H}$ of the code $\mathcal{C}$, and then successively adds all sums of not more than $d-2$ distinct rows of $\boldsymbol{H}$.

It is straightforward to invoke Theorem 3.10 for upper-bounding the stopping redundancy hierarchy $\rho_{\ell}(\mathcal{C})$ of a code - more precisely, in terms of adding all sums of at most $\ell-2$ rows to a given parity-check matrix. This result is stated below.

Theorem 3.11: Let $\mathcal{C}$ be a binary linear code with parameters $[n, k, d]$, with $d>3$. Then

$$
\rho_{\ell}(\mathcal{C}) \leq\left(\begin{array}{c}
n-k \\
1
\end{array}\right)+\ldots+\left(\begin{array}{c}
n-k \\
\ell-2
\end{array}\right) \text {. }
$$

Assume that one can identify a sub-code $\mathcal{B}$ of $\mathcal{C}^{\perp}$ with dual distance $d_{\mathcal{B}}^{\perp}$. Since the generators of a subcode form a subset of the generators of $\mathcal{C}^{\perp}$, one needs to apply the procedure of adding redundant rows leading to Theorem 3.1 or Theorems 3.83 .9 only to the basis vectors in $\mathcal{B}$ in order to ensure that the redundant matrix has stopping distance at least $d_{\mathcal{B}}^{\perp}$. This argument leads to the following result.

Theorem 3.12: Let $\Theta$ be the set of all sub-codes of the dual code $\mathcal{C}^{\perp}$ of a linear $[n, k, d]$ code $\mathcal{C}$ that have support weigh 11 $n$ and dual distance $\ell$. Furthermore, let the dimensions of the sub-codes in $\Theta$ be $K_{i}, i=1, \ldots,|\Theta|$, and define $K=\min _{i} K_{i}$. Then

$$
\rho_{\ell}(\mathcal{C}) \leq\left(\begin{array}{c}
K \\
1
\end{array}\right)+\ldots+\left(\begin{array}{c}
K \\
\ell-2
\end{array}\right)
$$

Proof: Let $\mathcal{C}_{1}$ be an $\left[n, n-K_{1}, \ell\right]$ code, and let its dual code $\mathcal{C}_{1}^{\perp}$ be a subcode of $\mathcal{C}^{\perp}$ of support $n$ and dimension $K_{1}$. From Equation (11), $\rho_{\ell}\left(\mathcal{C}_{1}\right) \leq\left(\begin{array}{c}K_{1} \\ 1\end{array}\right)+\ldots+\left(\begin{array}{c}K_{1} \\ \ell-2\end{array}\right)$. As $\mathcal{C}_{1}^{\perp}$ is a subcode of $\mathcal{C}^{\perp}$, the rows of the parity-check matrix of $\mathcal{C}_{1}$ are a subset of the rows of the parity-check matrix of $\mathcal{C}$. As a result, the upper bound also holds for $\rho_{\ell}(\mathcal{C})$.

Example 3.3: Let $\mathcal{G}$ be the $[24,12,8]$ extended Golay code. The code $\mathcal{G}$ is self-dual and contains a $[24,10,8]$ subcode. This code is unique [18], and has minimum dual distance 6 . Therefore

$$
\rho_{6}(\mathcal{G}) \leq\left(\begin{array}{c}
10 \\
1
\end{array}\right)+\left(\begin{array}{c}
10 \\
2
\end{array}\right)+\left(\begin{array}{c}
10 \\
3
\end{array}\right)+\left(\begin{array}{c}
10 \\
4
\end{array}\right)=385
$$

This bound is not tight as it by far exceeds the best known upper bound (found through extensive computer search [2]) on $\rho_{8}(\mathcal{G}) \leq 34$.

Example 3.4: Let $\mathcal{C}_{\mathrm{BCH}, 1}$ be the $[31,16,7] \mathrm{BCH}$ code. Since $\mathrm{BCH}$ codes are nested, this code is a subcode of a $[31,21,5]$ $\mathrm{BCH}$ code $\mathcal{C}_{\mathrm{BCH}, 2}$. Consequently, $\mathcal{C}_{\mathrm{BCH}, 2}^{\perp} \subseteq \mathcal{C}_{\mathrm{BCH}, 1}^{\perp}$, so that

$$
\rho_{5}\left(\mathcal{C}_{\mathrm{BCH}, 1}\right) \leq\left(\begin{array}{c}
10 \\
1
\end{array}\right)+\left(\begin{array}{c}
10 \\
2
\end{array}\right)+\left(\begin{array}{c}
10 \\
3
\end{array}\right)=175 .
$$

\section{Case Study: Parity-Check Matrices of Cyclic Form}

The lower and upper bounds on the stopping redundancy hierarchy, presented in the previous section, hold for all linear block codes. Unfortunately, as illustrated with several examples, these bounds tend to be very loose. As will be shown in this section, much tighter upper bounds on the stopping redundancy hierarchy of certain classes of codes can be obtained constructively, by focusing on special forms of parity-check matrices. In particular, we consider parity-check matrices of cyclic form. Upper bounds on the stopping distance of matrices in cyclic form also represent upper bounds on the stopping redundancy hierarchy of the codes. This claim is not true for lower bounds on the stopping distance hierarchy, although the derived bounds still offer valuable insight into the stopping set properties of matrices in cyclic form.

We start by specializing the bound of Section [II] to cyclic parity-check matrices, and then proceed with a case study of $\mathrm{BCH}$ codes and codes based on cyclic difference sets.

\section{A. Stopping Sets in Parity-Check Matrices of Cyclic Form}

We start this section by introducing cyclic codes and cyclic parity-check matrices.

Definition 4.1: Let $\mathcal{C}$ be an $[n, k, d]$ binary linear code. A code is called cyclic if a cyclic shift of a codeword $c \in \mathcal{C}$ is also a codeword. A (redundant) parity-check matrix of a cyclic code is said to be of cyclic form if it consists of $m$ cyclic shifts of one given codeword of the dual code, and provided that it has row-rank $n-k$.

Observe that parity-check matrices of cyclic form necessarily satisfy $n-k \leq m \leq n$. Also, note that a code can have a parity-check matrix of cyclic form without being cyclic - nevertheless, we focus our attention exclusively on cyclic codes. A standard form for $\boldsymbol{H}$ with $m=n-k$ cyclic row-shifts is shown below.

\footnotetext{
${ }^{1}$ The support weight of a sub-code of a code is defined as the number of positions for which at least one of the codewords of the sub-code is non-zero.
} 


$$
\boldsymbol{H}=\left(\begin{array}{cccccccc}
1 & \ldots & 1 & 0 & 0 & 0 & 0 & 0 \\
0 & 1 & \ldots & 1 & 0 & 0 & 0 & 0 \\
0 & 0 & 1 & \ldots & 1 & 0 & 0 & 0 \\
0 & 0 & 0 & 1 & \ldots & 1 & 0 & 0 \\
\vdots & & & \ddots & \ddots & & \ddots & 0 \\
0 & 0 & 0 & 0 & 0 & 1 & \ldots & 1
\end{array}\right)
$$

A binary cyclic code can be completely characterized by a normalized parity-check polynomial $h(x)=h_{0}+h_{1} x+h_{2} x^{2}+$ $\cdots+h_{k-1} x^{k-1}+x^{k}$, of degree $k$, for which $h_{i} \in \mathbb{F}_{2}, \forall i \in[0, k-1]$. In this case, a cyclic parity-check matrix $\boldsymbol{H}$ of the code can be constructed by setting the entry in the $i$-th row and $j$-th column to

$$
H[i, j]=\left.\frac{h^{(k-j+i \bmod n)}(x)}{(k-j+i \bmod n) !}\right|_{x=0}
$$

where $h^{(\zeta)}(x)$ denotes the $\zeta$-th derivative of $h, 1 \leq i \leq m, 1 \leq j \leq n$. This leads to a parity-check matrix of the form

$$
\boldsymbol{H}=\left(\begin{array}{ccccccc}
1 & h_{k-1} & \cdots & h_{1} & h_{0} & & \mathbf{0} \\
& \ddots & \ddots & & \ddots & & \\
\mathbf{0} & & 1 & h_{k-1} & \cdots & h_{1} & h_{0}
\end{array}\right) .
$$

Definition 4.2: Let $\mathcal{C}$ be a cyclic $[n, k, d]$ linear code. Partition the set of codewords of the dual code $\mathcal{C}^{\perp}$ into cyclic orbits of its codewords. More precisely, let $\mathcal{C}^{\perp}$ be the disjoint union $\bigcup_{c} G_{\boldsymbol{c}}$ in which $\boldsymbol{c} \in \mathcal{C}^{\perp}, G_{\boldsymbol{c}}=\left\{\pi^{i} \boldsymbol{c}, i=1, \ldots, n\right\}$, and where $\pi$ denotes the right-cyclic shift permutation of the symmetric group $S_{n}$. A designated element of $G_{c}$ is henceforth referred to as the cyclic orbit generator $(\operatorname{cog})$ of $G_{\boldsymbol{c}}$. Whenever apparent from the context, the reference to $G_{\boldsymbol{c}}$ will be omitted.

Note that, in general, the first row of a redundant parity-check matrix does not have to be defined by a parity-check polynomial. The first row can be any cog which has the property that a sufficient number of its cyclic shifts generates the dual code. In what follows, with a slight abuse of notation, we use $\boldsymbol{h}$ to denote such a $\operatorname{cog}$ (i.e. the first row of a cyclic parity-check matrix $\boldsymbol{H}$ ), and let $\boldsymbol{h}[i]$ denote its $i$-th entry.

We start by providing an intuitive explanation why (redundant) parity-check matrices of cyclic form have good stopping distance properties.

Definition 4.3: Without loss of generality, assume that the first row in a cyclic parity-check matrix $\boldsymbol{H}$ has a non-zero symbol in its first position. The span of the first row, denoted by $u$, is the largest value of the index $j$ for which $H[1, j]=1$. The zero-span $z$ of $\boldsymbol{h}$ represents the number of zeros trailing the last non-zero entry. Clearly, $u+z=n$.

Consider an arbitrary (redundant) cyclic parity-check matrix $\boldsymbol{H}$ of a code $\mathcal{C}$. The matrix consists of a non-zero $\operatorname{cog}$ and $m-1$ consecutive cyclic shifts thereof, where $n-k \leq m \leq n$. The matrix $\boldsymbol{H}$ has $i-1$ leading zeros and $z-i+1$ tailing zeros in row $i, 1 \leq i \leq m$. For any stopping set with largest column index $r \leq z+1$, the $r$-th row of the matrix has an entry "1" in column $r$, and zeros in all the remaining positions confined to $\{1,2, \ldots, r-1\}$. Therefore, the rows of $\boldsymbol{H}$ resolve all stopping sets of size $\sigma$ with support contained in the set $\{1,2, \ldots, z+1\}$. Similarly, for all stopping sets with coordinates confined to the set $\{n-z, n-z+1, \ldots, n\}$, let the smallest non-zero coordinate in the stopping set be indexed by $l$. In this case, the row indexed by $l-(n-z)$ has a " 1 " in the leftmost position $l$, and zeros in all remaining positions of the stopping set. Therefore, the rows of $\boldsymbol{H}$ also resolve all stopping sets of size $\sigma$ with support contained in the set $\{n-z, n-z+1, \ldots, n\}$.

It can also be easily seen that a redundant cyclic parity-check matrix with $m=n$ rows resolves all stopping sets with support confined to $z+1$ consecutive column indices. This straightforward analysis indicates that parity-check matrices of cyclic form may have good stopping distance properties. We explore these questions in more detail in the next section.

\section{B. Lower Bounds on the Stopping Distance of Cyclic Parity-Check Matrices}

We describe next how to bound the stopping distance of cyclic parity-check matrices by specializing the results of Section III

Proposition 4.4: The number of stopping sets of size $\sigma$ resolved by a cyclic (redundant) parity-check matrix with dimensions $m \times n, n-k \leq m \leq n$, is bounded from above by

$$
m\left|\Sigma_{\sigma, 1}\right|-\frac{2}{m} \cdot \sum_{\kappa=1}^{m-1}(m-\kappa)\left|\Sigma_{\sigma, 1} \cap \Sigma_{\sigma,\left((1+\kappa) \bmod ^{*} m\right)}\right| .
$$

The notation $\bmod ^{*}$ is reserved for the modulo function, for which $m \bmod ^{*} m$ equals $m$, rather than zero.

In the proposition, it is tacitly assumed that the rows of the parity-check matrix are arranged in such a way that the row indexed by $\left((\kappa+1) \bmod ^{*} m\right)$ is the $\kappa$-th cyclic shift of the first row.

The result of Proposition 4.4 is a specialization of Equation (3). In a cyclic parity-check matrix, each row resolves the same number of stopping sets as the positions of the resolved stopping sets are cyclic shifts of each other. Furthermore, the number 
of stopping sets simultaneously resolved by two rows indexed by $i$ and $j, i \neq j$, only depends on the their mutual cyclic shift distance $\kappa$.

Let the set $X Y_{\kappa}(\boldsymbol{h})$ denote all pairs of positions $(a, a+\kappa) \bmod ^{*} n$ in $\boldsymbol{h}, 1 \leq a \leq n$, with entry $X$ at position $a \bmod ^{*} n$ and entry $Y$ at position $a+\kappa \bmod ^{*} n, X, Y \in\{Z, O\}$. For parity-check matrices of cyclic form, the cardinalities of these sets equal the intersection numbers given in Definition 3.5, i.e. $\left|X Y_{\kappa}(\boldsymbol{h})\right|=\left|X_{a \bmod ^{*} n} \cap Y_{a+\kappa} \bmod ^{*} n\right|, \forall a \in\{1, \ldots, n\}$. If clear from the context, we omit the reference to $h$.

Lemma 4.5: Let $\delta[k]$ be the indicator function, such that $\delta[k]=1$ iff $k=0$. The number of stopping sets resolved jointly by two rows of a cyclic parity-check matrix $\kappa$ cyclic shifts apart equals

$$
\left|\Sigma_{\sigma, 1} \cap \Sigma_{\sigma,\left((\kappa+1) \bmod ^{*} m\right)}\right|=\left|O O_{\kappa}\right| \cdot\left(\begin{array}{c}
\left|Z Z_{\kappa}\right| \\
\sigma-1
\end{array}\right)+\left|O Z_{\kappa}\right| \cdot\left|Z O_{\kappa}\right| \cdot\left(\begin{array}{c}
\left|Z Z_{\kappa}\right| \\
\sigma-2
\end{array}\right),
$$

where

$$
\begin{gathered}
\left|O O_{\kappa}\right|=\sum_{i=1}^{n}\left\lfloor\frac{1}{2} \sum_{j=1}^{n} h[j](\delta[j-i]+\delta[j-i-\kappa])\right\rfloor, \\
\left|Z Z_{\kappa}\right|=\sum_{i=1}^{n}\left\lfloor\frac{1}{2} \sum_{j=1}^{n}(1-h[j])(\delta[j-i]+\delta[j-i-\kappa])\right\rfloor, \\
\left|Z O_{\kappa}\right|=\left|O Z_{\kappa}\right|=\sum_{i=1}^{n}\left[\frac{1}{2}\left(\sum_{j=1}^{n} h[j](\delta[j-i]+\delta[j-i-\kappa])\right) \cdot\left|\sum_{j=1}^{n}(h[j](\delta[j-i]+\delta[j-i-\kappa]))-2\right|\right],
\end{gathered}
$$

and

$$
\left|\Sigma_{\sigma, 1}\right|=\left(\begin{array}{c}
n-\sum_{j=1}^{n} h[j] \\
\sigma-1
\end{array}\right) \cdot \sum_{j=1}^{n} h[j] .
$$

Note that in all equations above it is assumed that $\left(\begin{array}{l}i \\ j\end{array}\right)=0$ for $i<j$.

The expressions in Lemma 4.5 are derived by simple counting arguments, details of which are omitted. Although the expressions above cannot be used to directly characterize the stopping redundancy hierarchy, they represent a useful tool for evaluating the stopping distance properties of cyclic parity-check matrices, as illustrated by the examples that follow.

Example 4.1: Consider the class of quadratic residue $(\mathrm{QR})$ codes with prime length $n=3(\bmod 4)$. Let the redundant parity-check matrix be of cyclic form, and let the first row of the parity-check matrix be defined by the idempotent of the code $\mathcal{Q}^{\perp}$ (defined in [11]), $I_{q}(x)=1+\sum_{\nu \in \mathcal{N}} x^{\nu}$. Here, $\mathcal{N}$ denotes the set of quadratic non-residues in $\mathbb{F}_{n} 2$. The cyclic parity-check matrix generated by $m \leq n$ cyclic shifts of the idempotent has $S_{\sigma, 2}$ stopping sets resolved by all pairs of rows, where

$$
S_{\sigma, 2}=\left(\begin{array}{c}
m \\
2
\end{array}\right) \cdot \frac{n+1}{4} \cdot\left(\left(\begin{array}{c}
\frac{n-3}{4} \\
\sigma-1
\end{array}\right)+\frac{n+1}{4}\left(\begin{array}{c}
\frac{n-3}{4} \\
\sigma-2
\end{array}\right)\right) .
$$

Be reminded that $S_{\sigma, 2}$ denotes the number of stopping sets resolved by all pairs of two rows (cf. Section ЩII-A). As a consequence, for such a cyclic parity-check matrix to have stopping distance at least $\ell$, its number of rows $\mu_{\ell}$ must satisfy the following inequality

$$
\mu_{\ell}(\mathcal{C}) \geq \max _{\sigma<\ell}\left\lceil\frac{\left(\begin{array}{c}
n \\
\sigma
\end{array}\right)-M}{\frac{n+1}{2}\left(\frac{n-1}{\sigma-1}\right)-M}\right\rceil,
$$

where

$$
M=\frac{n+1}{4} \cdot\left[\left(\begin{array}{c}
\frac{n-3}{4} \\
\sigma-1
\end{array}\right)+\frac{n+1}{4} \cdot\left(\begin{array}{c}
\frac{n-3}{4} \\
\sigma-2
\end{array}\right)\right] .
$$

A detailed derivation of this result can be found in Appendix IV Numerical values for the numbers $\mu_{\ell}$, for three chosen QR codes, are given in Table \. The bounds in the table imply that for the $[47,24,11]$ code, the stopping distance of any redundant parity-check matrix of cyclic form, defined by the idempotent, cannot exceed seven.

Example 4.2: As a second example, consider the class of cyclic difference set (CDS) codes [11], [19], [20], formally defined below.

Definition 4.6: Assume that all calculations are performed modulo $n$. A $(n, k, \lambda) \operatorname{CDS}$ is a set $Q=\left\{d_{0}, d_{1}, \ldots, d_{J-1}\right\}$ of $J$ integers with the property that any non-zero integer $i \leq n$, is a difference of two elements in $Q$ and that there exist exactly $\lambda$ ways to choose these two elements of $Q$.

\footnotetext{
${ }^{2}$ For more details regarding QR codes, the interested reader is referred to Section 16 of [11].
} 
TABLE I

LOWER BOUNDS ON $\mu_{\ell}$ FOR PARITY-CHECK MATRICES OF CYCLIC FORM GENERATED FROM THE IDEMPOTENT OF QR CODES.

\begin{tabular}{|c|cccccccc|}
\hline & $\mu_{4}$ & $\mu_{5}$ & $\mu_{6}$ & $\mu_{7}$ & $\mu_{8}$ & $\mu_{9}$ & $\mu_{10}$ & $\mu_{11}$ \\
\hline$[23,12,7]$ Golay & 4 & 6 & 10 & 19 & & & & \\
{$[31,16,7]$ BCH } & 4 & 6 & 9 & 17 & & & & \\
{$[47,24,11]$ QR } & 4 & 6 & 9 & 15 & 27 & 55 & 117 & 265 \\
\hline
\end{tabular}

For a CDS, the sets $Q_{r}=\left\{d_{0}+r, d_{1}+r, \ldots, d_{J-1}+r\right\}$ for all $r=0, \ldots, n-1$, form a cyclic $2-(n, k, \lambda)$ block design [21]. According to [19], a CDS code of length $n$ is characterized by a polynomial $z(x)=\sum_{i=0}^{k-1} x^{d_{i}}$ that gives rise to a parity-check polynomial $h(x)=\operatorname{gcd}\left(z(x), x^{n}+1\right)$.

For this particular case of cyclic codes, one can show that if $h(x)=z(x)$, then

$$
\left|O O_{\kappa}\right|=\lambda, \quad\left|O Z_{\kappa}\right|=\left|Z O_{\kappa}\right|=k-\lambda, \quad\left|Z Z_{\kappa}\right|=n-2 k+\lambda, \forall \kappa>0,
$$

and that

$$
S_{\sigma, 2}=\left(\begin{array}{c}
m \\
2
\end{array}\right)\left(\lambda\left(\begin{array}{c}
n-2 k+\lambda \\
\sigma-1
\end{array}\right)+(k-\lambda)^{2}\left(\begin{array}{c}
n-2 k+\lambda \\
\sigma-2
\end{array}\right)\right) .
$$

As a result,

$$
\mu_{\ell} \geq \max _{\sigma<\ell}\left\lceil\frac{\left(\begin{array}{l}
n \\
\sigma
\end{array}\right)-M}{k \cdot\left(\begin{array}{l}
n-k \\
\sigma-1
\end{array}\right)-M}\right\rceil,
$$

where

$$
M=\lambda \cdot\left(\begin{array}{c}
n-2 k+\lambda \\
\sigma-1
\end{array}\right)+(k-\lambda)^{2} \cdot\left(\begin{array}{c}
n-2 k+\lambda \\
\sigma-2
\end{array}\right),
$$

and where $\mu_{\ell}$ is defined as in the previous example.

Consider the class of Singer difference sets [21], with parameters

$$
(n, k, \lambda)=\left(\frac{q^{a+1}-1}{q-1}, \frac{q^{a}-1}{q-1}, \frac{q^{a-1}-1}{q-1}\right),
$$

where we specialize $a=2$, and $q=2^{s}$. The resulting difference set has parameters $(n, k, \lambda)=\left(2^{s}\left(2^{s}+1\right)+1,2^{s}+1,1\right)$, and leads to a $\left[2^{s}\left(2^{s}+1\right)+1,2^{s}\left(2^{s}+1\right)-3^{2}, 2^{2}+2\right]$ CDS code, for which, if $h(x)=z(x)$ as defined above,

$$
\mu_{\ell} \geq \max _{\sigma<\ell}\left\lceil\frac{\left(\begin{array}{c}
2^{2 s}+2^{s}+1 \\
\sigma
\end{array}\right)-\left(\begin{array}{c}
2^{2 s}-2^{s} \\
\sigma-1
\end{array}\right)-2^{2 s}\left(\begin{array}{c}
2^{2 s}-2^{s} \\
\sigma-2
\end{array}\right)}{\left(2^{s}+1\right) \cdot\left(\begin{array}{c}
2^{2 s} \\
\sigma-1
\end{array}\right)-\left(\begin{array}{c}
2^{2 s}-2^{s} \\
\sigma-1
\end{array}\right)-2^{2 s} \cdot\left(\begin{array}{c}
2^{2 s}-2^{s} \\
\sigma-2
\end{array}\right)}\right\rceil .
$$

The results presented in this section characterized the smallest number of rows in a cyclic parity-check matrix needed for resolving all (or a certain number of) stopping sets of a given size. To obtain upper bounds on these numbers, we resort to constructive methods that rely on identifying good choices for a cog defining the parity-check matrix. Our cog selection procedure, along with a comparison of all constructive redundancy parity-check design methods discussed, is presented in the next section.

\section{Upper Bounds on the Stopping Distance of Cyclic Parity-Check Matrices}

In what follows, we focus on deriving upper bounds on the stopping distance of cyclic parity-check matrices of the Golay code and BCH codes. We review several known approaches for constructing redundant parity-check matrices for Hamming codes with a prescribed number of stopping sets of a given size, and then compare them to those based on optimizing cyclic parity-check matrices. For Hamming codes, the number of stopping sets of a full-rank, non-redundant parity-check matrix was derived in analytical form in [3]. We observed that this number does not depend on the form of the parity-check matrix. However, if redundant parity-check matrices are used, the number of stopping sets clearly depends on the chosen form of the matrix.

As will be shown, for a given stopping set distribution, cyclic parity-check matrices for the Golay and BCH codes offer row-redundancies comparable to those achievable by the best known methods described in [2] and [10], respectively. For the general class of $\mathrm{BCH}$ codes, we first provide a new method for constructing redundant parity-check matrices with prescribed stopping distance, by extending the work in [10]. We then compare these findings with the stopping redundancy parameters obtained from parity-check matrices of cyclic form. 
1) The Binary Golay Code: To evaluate the performance of cyclic parity-check matrices of the $[23,12,7]$ Golay code $\mathcal{G}$, the 506 codewords of minimum weight eight in the dual $[23,11,8]$ code $\mathcal{G}^{\perp}$ are grouped into cog orbits. The codewords of weight eight in $\mathcal{G}^{\perp}$ also belong to the code $\mathcal{G}$, and can be arranged into 22 cog orbits with 23 codewords each.

Different choices for a cyclic parity-check matrix-defining cog result in different stopping distance bounds. We concentrate on two cogs that offer the best and the worst stopping distance properties among all investigated cogs, and refer to them by the subscripts $\mathrm{A}$ and D.

The cog indexed by A has the following octal representation $\operatorname{cog}_{[23,12], \mathrm{A}}=[21213500]$, with the most significant bit on the left side. Its polynomial representation is $1+x^{4}+x^{6}+x^{10}+x^{12}+x^{13}+x^{14}+x^{16}$. The cog indexed by $\mathrm{D}$ has the

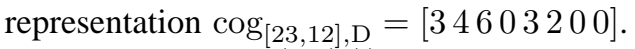

Upper bounds $U\left(\rho_{j}(\mathcal{C})\right)$ on the stopping redundancy hierarchy obtained from cogs $\operatorname{cog}_{[23,12], \mathrm{A}}, \operatorname{cog}_{[23,12], \mathrm{B}}$, and $\operatorname{cog}_{[23,12], \mathrm{D}}$ of the parity-check matrix of the $[23,12,7]$ Golay code and the general upper bounds obtained from Theorem 3.9 with $\epsilon=10^{-3}$ and from Equation (10) are listed in Table प]

TABLE II

UPPER BOUNDS ON THE STOPPING REDUNDANCY HIERARCHY OF THE $[23,12,7]$ GOLAY CODE.

\begin{tabular}{|c|c|c|c|c|}
\hline & $U\left(\rho_{4}(\mathcal{C})\right)$ & $U\left(\rho_{5}(\mathcal{C})\right)$ & $U\left(\rho_{6}(\mathcal{C})\right)$ & $U\left(\rho_{7}(\mathcal{C})\right)$ \\
\hline $\operatorname{cog}_{23,12, \mathrm{~A}}$ & $n-k$ & 16 & 18 & 23 \\
$\operatorname{cog}_{23,12, \mathrm{~B}}$ & 13 & 15 & 19 & 23 \\
$\operatorname{cog}_{23,12, \mathrm{D}}$ & $n-k$ & 16 & 21 & $>23$ \\
\hline Minimum & $n-k$ & 15 & 18 & 23 \\
\hline Upper bound acc. to Theorem 3.9] $\epsilon=10^{-3}$ & 39 & - & - & - \\
\hline Upper bound acc. to Eq. 10 & 1023 & 1023 & 1023 & 1023 \\
\hline
\end{tabular}

As can be seen from Table $\Pi$, the stopping redundancy hierarchy of the Golay code is bounded as

$$
\rho_{4}(\mathcal{C}) \leq n-k, \rho_{5}(\mathcal{C}) \leq 15, \rho_{6}(\mathcal{C}) \leq 18, \rho_{7}(\mathcal{C}) \leq 23
$$

In addition, from the same table it can be seen that the bound in Equation (10) is extremely loose - almost ten-fold reductions are possible when using the constructive cyclic matrix design approach.

2) The Hamming Codes: Several approaches for generating parity-check matrices of Hamming codes that resolve correctable erasure patterns up to a given size were recently described by Weber and Abdel-Ghaffar [9] and Hollmann and Tolhuizen [10]. In [9], the authors introduced the notion of three-erasure correcting parity-check collections, capable of resolving the largest possible number of erasure patterns of weight three. These results were extended in [10], where generic $(\bar{m}, \bar{\sigma})$ erasure correcting sets $\boldsymbol{A}_{\bar{m}, \bar{\sigma}}$ were defined as

$$
\left\{\boldsymbol{a}=\left(a_{1}, a_{2}, \ldots, a_{\bar{m}}\right) \mid a_{1}=1, \operatorname{wt}(\boldsymbol{a}) \leq \bar{\sigma}\right\} .
$$

In this context, if $\boldsymbol{H}$ represents a parity-check matrix of dimension $\bar{m} \times \bar{\sigma}$, the collection of parity-checks $\left\{\boldsymbol{a} \boldsymbol{H} \mid \boldsymbol{a} \in \boldsymbol{A}_{\bar{m}, \bar{\sigma}}\right\}$ resolves all correctable erasure patterns up to size $\bar{\sigma}$.

For the class of $\left[2^{s}-1,2^{s}-s-1, d=3\right]$ Hamming codes, according to [10], we first construct parity-check matrices that are generic $(\bar{m}, \bar{\sigma})$ erasure correcting sets, and then compare the stopping set distribution of these matrices with that of optimized cyclic parity-check matrices. As for Hamming codes $\rho_{2}(\mathcal{C})=\rho_{3}(\mathcal{C})=n-k$ and $d=3$, our comparison is performed with respect to the number of unresolved stopping sets of size $\sigma=3$, for several fixed values of $m$.

Assume that the parity-check matrix of a Hamming code is given by a row vector over the finite field $\mathbb{F}_{n+1}, n=2^{n^{\prime}}-1, n^{\prime}>$ 0 , namely

$$
\boldsymbol{H}_{\mathrm{h}}=\left(\begin{array}{llll}
\alpha^{0 \cdot b} & \alpha^{1 \cdot b} & \ldots & \alpha^{(n-1) b}
\end{array}\right),
$$

where $\alpha$ denotes a primitive element of the underlying field. Henceforth, we set $b=1$. In order to form a binary parity-check matrix for the Hamming code, the elements of $\boldsymbol{H}_{\mathrm{h}}$ are represented as binary vectors over the vector space $\mathbb{F}_{2}^{\log _{2}(n+1)}$, i.e. each element $\alpha^{f}, f \in\{0, \ldots, n-1\}$, is described by a binary column vector. Let us refer to this parity-check matrix as the standard parity-check matrix for Hamming codes and denote it by $\boldsymbol{H}_{\mathrm{h}, \mathrm{s}}$.

Start by constructing a generic erasure correcting set $\boldsymbol{A}_{\bar{m}, \bar{\sigma}}$ with $\bar{m}=\log _{2}(n+1)$ and $\bar{\sigma}=3$. By applying the generic erasure set construction to $\boldsymbol{H}_{\mathrm{h}, \mathrm{s}}$, we arrive at a redundant parity-check matrix of the code denoted by $\boldsymbol{H}_{\mathrm{h}, \mathrm{g}}$. This matrix has

$$
m^{\star}=\sum_{i=0}^{\bar{\sigma}-1}\left(\begin{array}{c}
\bar{m}-1 \\
i
\end{array}\right)=\log _{2}(n+1)+\left(\begin{array}{c}
\log _{2}(n+1)-1 \\
2
\end{array}\right)
$$

rows. Note that the only unresolved stopping sets of size three in such a parity-check matrix are the actual codewords of the Hamming code. We then proceed by constructing redundant parity-check matrices of cyclic form, obtained from $m^{\star}$ consecutive cyclic shifts of a minimum weight codeword of the dual code. Table $\amalg$ and Table IV show the number of unresolved stopping 
sets of size three in the redundant parity-check matrices described above, for $n=63$ and $n=127$. The cog vectors of the two cyclic parity-check matrices are

$$
\operatorname{cog}_{[63,57]}=[414247507113354653740],
$$

and

$$
\operatorname{cog}_{[127,120]}=[1046135330146516366412575121561770357131100],
$$

respectively. The vectors are listed in octal form, with the most significant bit on the left hand side.

TABLE III

Number of UnRESOLVEd StopPing SETS $(\sigma=3)$ IN THE $[63,57,3]$ HAMMing COdE.

\begin{tabular}{|c|c|c|}
\hline \multirow{2}{*}{$m$} & \multicolumn{2}{|c|}{ Number of unresolved stopping sets, $\sigma=3$} \\
\cline { 2 - 3 } & Parity-check matrix in [10] & Cyclic parity-check matrix \\
\hline $6=n-k$ & 2261 & 2261 \\
16 & 651 & 655 \\
17 & & 653 \\
18 & & 651 \\
\hline
\end{tabular}

TABLE IV

Number of UNRESOLVED STOPPING SETS $(\sigma=3)$ of THE $[127,120,3]$ HAMming Code.

\begin{tabular}{|c|c|c|}
\hline \multirow{2}{*}{$m$} & \multicolumn{3}{|c|}{ Number of unresolved stopping sets, $\sigma=3$} \\
\cline { 2 - 3 } & Parity-check matrix in [10] & Cyclic parity-check matrix \\
\hline $7=n-k$ & 11970 & 11970 \\
22 & 2667 & 2672 \\
26 & & 2667 \\
\hline
\end{tabular}

As one can observe from Tables [II] and [V] cyclic parity-check matrices can achieve almost identical performance to the one offered by redundant parity-check matrices specialized for the Hamming codes. For example, there are 11970 stopping sets of size $\sigma=3$ in a full-rank parity-check matrix of the [127,120,3] Hamming code. This number can be reduced to 2667 stopping sets only, if one uses 22 rows of the construction described in [10], or 26 rows, when using parity-check matrices of cyclic form. These findings motivate a further study of redundant cyclic parity-check matrices, and we describe some illustrative examples pertaining to the family of $\mathrm{BCH}$ codes in the next section.

3) Cyclic Parity-Check Matrices of Double-and Triple-Error Correcting BCH Codes: We present constructive upper bounds on the stopping redundancy hierarchy of the $[127,113,5]$ double-error correcting $\mathrm{BCH}$ code, and the $[31,16,7]$ triple-error correcting BCH code. Similarly as for the Golay code, we only consider minimum-weight codewords of the dual code for the purpose of generating redundant parity-check matrices in cyclic form [22]. In particular, we focus on four selected cogs, indexed by A through $\mathrm{D}$, and analyze their underlying parity-check matrices in detail.

The dual of the $[127,113,5] \mathrm{BCH}$ code has 4572 codewords of minimum weight 56 , which can be separated into $36 \mathrm{cog}$ orbits. The octal representations of four different cogs used in our constructions are listed below:

$$
\begin{aligned}
\operatorname{cog}_{[127,113], \mathrm{A}} & =[1764030654454075045476516160204265242440056], \\
\operatorname{cog}_{[127,113], \mathrm{B}} & =[1724250261215411115261072125516140465414274], \\
\operatorname{cog}_{[127,113], \mathrm{C}} & =[1752655336461312642107117040240254030452242], \\
\operatorname{cog}_{[127,113], \mathrm{D}} & =[1751703125267346502102070365406122101430644] .
\end{aligned}
$$

The dual of the $[31,16,7] \mathrm{BCH}$ code has 465 codewords of minimum weight eight, which can be partitioned into $15 \operatorname{cog}$ orbits. Four representative cogs, indexed by $A$ through $D$, are listed below.

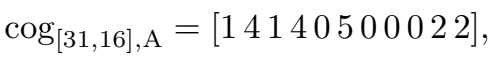

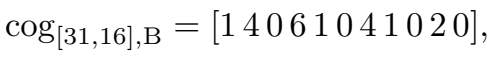

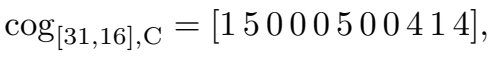

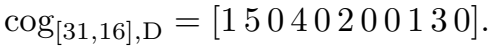


We now compare the stopping distance properties of parity-check matrices of cyclic form with that of a novel construction of redundant parity-check matrices generalizing the method in [10]. In a nutshell, the construction exploits the fact that $\mathrm{BCH}$ codes are nested, and that they are subcodes of Hamming codes. Redundant parity-check equations are chosen in two different steps: in the first step, they are selected to eliminate stopping sets corresponding to certain codewords of the Hamming code, while in the second step they are chosen to eliminate stopping sets that do not correspond to codewords in the Hamming code. A detailed description of this scheme is given in Appendix V For simplicity, we refer to it as the generalized HT (Hollmann-Tolhuizen) construction.

We next compare the stopping distances of various redundant parity-check matrices of the aforementioned $\mathrm{BCH}$ codes. The smallest values found by this comparison represent constructive upper bounds on $\rho_{\ell}(\mathcal{C}), \ell \leq d$, of $\mathrm{BCH}$ codes.

Table $\nabla$ lists the number of rows in a redundant parity-check matrix needed to achieve a given stopping distance, for matrices constructed by the generalized HT method, the upper bound from [10], and a bound obtained from cyclic parity-check matrices. In addition, the upper bound from [2], given in Equation (10), is also shown. All results pertain to the [127, 113, 5] BCH code.

TABLE V

UPPER BOUNDS ON $\rho_{\ell}(\mathcal{C}), \ell \leq 5$.

\begin{tabular}{|c|c|c|c|c|}
\hline \multirow{2}{*}{ Code } & \multicolumn{4}{|c|}{$U\left(\rho_{4}(\mathcal{C})\right)$} \\
\cline { 2 - 5 } & Cyclic & Gen. HT & {$[10]$} & General [2] \\
\hline$[127,113,5]$ & 20 & 96 & 92 & 469 \\
\hline \hline \multirow{3}{*}{ Code } & \multicolumn{4}{|c|}{$U\left(\rho_{5}(\mathcal{C})\right)$} \\
\cline { 2 - 5 } & Cyclic & Gen. HT & {$[10]$} & General [2] \\
\hline$[127,113,5]$ & 34 & 229 & 378 & 469 \\
\hline
\end{tabular}

The values presented for cyclic codes are obtained by searching for the best cog orbit, with cogs corresponding to minimum weight codewords. The cog indexed by $A$ has the "best" stopping set properties, when both the numbers of stopping sets of size three and four are considered. Stopping distances of all four described cogs are given in Table VI.

As a consequence, for the $[127,113,5] \mathrm{BCH}$ code $\mathcal{B}_{[127,113,5]}$ one has

$$
\rho_{4}\left(\mathcal{B}_{[127,113,5]}\right) \leq 20, \rho_{5}\left(\mathcal{B}_{[127,113,5]}\right) \leq 34 .
$$

Table $\mathrm{V}$ shows that the general bounds derived in [2] are very loose. Although the approach from [10] provides tighter bounds for this class of codes, it is still significantly outperformed by both the generalized HT method, as well as the cyclic (redundant) parity-check matrix construction.

TABLE VI

UPPER BOUNDS ON THE STOPPING REDUNDANCY HIERARCHY OF THE $[127,113,5]$ BCH CODE.

\begin{tabular}{|c|c|c|}
\hline & $U\left(\rho_{4}(\mathcal{C})\right)$ & $U\left(\rho_{5}(\mathcal{C})\right)$ \\
\hline $\operatorname{cog}_{127,113, \mathrm{~A}}$ & 20 & 34 \\
$\operatorname{cog}_{127,113, \mathrm{~B}}$ & 22 & 34 \\
$\operatorname{cog}_{127,113, \mathrm{C}}$ & 23 & 46 \\
$\operatorname{cog}_{127,113, \mathrm{D}}$ & 22 & 45 \\
\hline Minimum & 20 & 34 \\
\hline Eq. (10) & 469 & 469 \\
\hline
\end{tabular}

The results of a similar analysis performed on the $[31,16,7] \mathrm{BCH}$ code $\mathcal{B}_{[31,16,7]}$ are shown in Table VII As can be seen

TABLE VII

UPPER BOUNDS ON THE STOPPING REDUNDANCY HIERARCHY OF THE $[31,16,7]$ BCH CODE.

\begin{tabular}{|c|c|c|c|c|}
\hline & $U\left(\rho_{4}(\mathcal{C})\right)$ & $U\left(\rho_{5}(\mathcal{C})\right)$ & $U\left(\rho_{6}(\mathcal{C})\right)$ & $U\left(\rho_{7}(\mathcal{C})\right)$ \\
\hline $\operatorname{cog}_{31,16, \mathrm{~A}}$ & $n-k=15$ & 18 & 19 & 21 \\
$\operatorname{cog}_{31,16, \mathrm{~B}}$ & $n-k=15$ & 16 & 20 & 22 \\
$\operatorname{cog}_{31,16, \mathrm{C}}$ & $n-k=15$ & $n-k=15$ & 20 & 28 \\
$\operatorname{cog}_{31,16, \mathrm{D}}$ & $n-k=15$ & 16 & 21 & 26 \\
\hline Minimum & $n-k=15$ & $n-k=15$ & 19 & 21 \\
\hline Upper bound acc. to Theorem 3.9 $\epsilon=10^{-3}$ & 45 & - & - & - \\
\hline Upper bound acc. to Eq. (10, 112$)$ & 91 & 175 & 4943 & 4943 \\
\hline
\end{tabular}

from the table, the stopping redundancy hierarchy of the $[31,16,7] \mathrm{BCH}$ codes is upper bounded as

$$
\rho_{4}\left(\mathcal{B}_{[31,16,7]}\right)=15, \rho_{5}\left(\mathcal{B}_{[31,16,7]}\right)=15, \rho_{6}\left(\mathcal{B}_{[31,16,7]}\right) \leq 19, \rho_{7}\left(\mathcal{B}_{[31,16,7]}\right) \leq 21 .
$$




\section{The Automorphism Redundancy}

One important observation motivates our subsequent study of stopping redundancy properties of cyclic (redundant) paritycheck matrices. It is the simple fact that using a collection of cyclically shifted rows in $\boldsymbol{H}$ for resolving a stopping set has the same effect as using only one of these rows and then cyclically shifting the received codeword.

In general, the same observation is true for parity-check matrices that consist of rows that represent different images of one given row-vector under a set of coordinate permutations. Such coordinate permutations must necessarily preserve the codeword structure, i.e. they must correspond to automorphisms of the code. Furthermore, if properly chosen, such automorphisms may be used to relocate the positions of the erasures in the received codeword to coordinates that do not correspond to stopping sets.

We propose to use this observation in order to construct parity-check matrices of codes that, jointly with a new decoding technique, allow the edge-removal algorithm to avoid detrimental effects of stopping sets on its performance. For this purpose, we first recall the definition of the automorphism group of a code.

Definition 5.1: ([11, Ch. 8]) The set of permutations which send a code $\mathcal{C}$ into itself, i.e. permutations that map codewords into (possibly different) codewords, are called the automorphism group of the code $\mathcal{C}$, and are denoted by $\operatorname{Aut}(\mathcal{C})$.

It is straightforward to see that $\operatorname{Aut}(\mathcal{C})=\operatorname{Aut}\left(\mathcal{C}^{\perp}\right)$, a fact that we exploit in our subsequent derivations.

Decoding procedures that use information about the automorphism group of a code have a long history [11, Ch. 16]. Such procedures are known as permutation decoding algorithms and they are traditionally restricted to decoding of messages transmitted over binary symmetric channels (BSCs) 3 . Within the framework of permutation decoding, a codeword $c \in \mathcal{C}$, corrupted by an error vector $\boldsymbol{e}=\left[e_{0}, e_{1}, \ldots, e_{n-1}\right]$ of weight less than or equal to $t$, where $2 t+1 \leq d$, is subjected to the following process. First, a parity-check matrix of the code of the form $\boldsymbol{H}=[\boldsymbol{A} \mid \boldsymbol{I}]$, along with the corresponding generator matrix $\boldsymbol{G}=\left[\boldsymbol{I} \mid \boldsymbol{A}^{\mathrm{T}}\right]$, is chosen. Here, $\boldsymbol{I}$ is used to denote the identity matrices of order $n-k$ and $k$, respectively. Then, the syndrome of the received vector $\boldsymbol{y}=\boldsymbol{c}+\boldsymbol{e}, \boldsymbol{z}=\boldsymbol{H} \boldsymbol{y}^{\mathrm{T}}$, is computed. If the weight of $\boldsymbol{z}$ is greater than $t$, the vector $\boldsymbol{y}$ is permuted according to a randomly chosen automorphism. This process is repeated until either all automorphisms are tested or until the syndrome has weight less than or equal to $t$. In the former case, the decoder declares an error. In the latter case, all decoding errors are provably confined to parity-check positions, so that decoding terminates by recovering the uncorrupted information symbols.

For the purpose of permutation decoding, one would like to identify the smallest set of automorphisms that moves any set of not more than $t$ positions in $\{0, \ldots, n-1\}$ into the parity-check positions $\{k, k+1, \ldots, n-1\}$ of $[\boldsymbol{A} \mid \boldsymbol{I}]$.

Definition 5.2: ([24]) If $\mathcal{C}$ is a $t$-error correcting code with an information bit index set $\mathcal{I}$ and a parity-check index set $\mathcal{P}$, then a $\mathbf{P D}(\mathcal{C})$-set (permutation decoding set of $\mathcal{C}$ ) is a set $S$ of automorphisms of $\mathcal{C}$ such that every $t$-set of coordinate positions is moved by at least one member of $S$ into the check-positions $\mathcal{P}$. For $s \leq t$, an $\mathbf{s - P D}(\mathcal{C})$-set is a set of automorphisms of $\mathcal{C}$ such that every $b$-set of coordinate positions, for all $b \leq s$, is moved by at least one member of $S$ into $\mathcal{P}$.

Throughout the remainder of this paper, we will be concerned with PD and s-PD sets of smallest possible size, and we simply refer to them as PD sets. Clearly, PD and s-PD sets may not exist for a given code, and complete or partial knowledge about PD sets is available for very few codes [24]. Nevertheless, even this partial information can be used to derive useful results regarding the analogues of PD sets for iterative decoders operating on stopping sets.

We introduce next the notion of a Stopping Automorphism Group Decoding (SAD) set, a generalization of the notion of a PD set for the edge-removal iterative decoder. We then proceed to relate SAD sets to both PD sets and to the stopping redundancy hierarchy of a code.

Definition 5.3: Let $\boldsymbol{H}$ be a parity-check matrix of an error-correcting code $\mathcal{C}$ with minimum distance $d$. A $\mathbf{S A D}(\boldsymbol{H}) \operatorname{set}$ of $\boldsymbol{H}$ is the smallest set $S$ of automorphisms of $\mathcal{C}$ such that every $b$-set of coordinate positions, $1 \leq b \leq d-1$, is moved by at least one member of $S$ into a set of positions that do not correspond to a stopping set of $\boldsymbol{H}$. Similarly, if $s \leq d-1$, an s-SAD $(\boldsymbol{H})$-set is the smallest set of automorphisms of $\mathcal{C}$ such that every $b$-set of coordinate positions, for all $b \leq s$, is moved by at least one member of $S$ into positions that do not correspond to a stopping set in $\boldsymbol{H}$. Without loss of generality, we assume that an s-SAD $(\boldsymbol{H})$-set contains the identity permutation. For a given code $\mathcal{C}$, we also define

$$
\begin{aligned}
\mathbf{S}_{s}^{\star}(\mathcal{C}) & =\min _{\boldsymbol{H}(\mathcal{C})}|\mathbf{s}-\mathbf{S A D}(\boldsymbol{H}(\mathcal{C}))|, \\
\mathbf{S}^{\star}(\mathcal{C}) & =\min _{\boldsymbol{H}(\mathcal{C})}|\mathbf{S A D}(\boldsymbol{H}(\mathcal{C}))|,
\end{aligned}
$$

and refer to $\mathbf{S}_{s}^{\star}(\mathcal{C})$ and $\mathbf{S}^{\star}(\mathcal{C})$ as to the s-automorphism redundancy and automorphism redundancy of $\mathcal{C}$.

For a given code and parameter $\mathbf{s}$, a s-SAD set may not exist. This is a consequence of the fact that there may be no automorphisms that move all arbitrary collections of not more than $s$ coordinates into positions that do not correspond to a stopping set in one given parity-check matrix. But whenever such sets exist, they can be related to the stopping redundancy hierarchy and PD sets of the code. First, it is straightforward to show that for all $1 \leq s \leq d-1$, one has $\mathbf{S}_{s}^{\star}(\mathcal{C}) \leq|\mathbf{s}-\mathbf{P D}(\mathcal{C})|$, whenever such sets exist. This follows from considering parity-check matrices in systematic form, and from the Singleton

\footnotetext{
${ }^{3}$ Recently, permutation decoders were also used for decoding of messages transmitted over the AWGN channel [23], although not for the purpose of finding error-free information sets nor for the purpose of eliminating pseudocodewords such as stopping sets.
} 
bound, which asserts that for any linear code $d-1 \leq n-k$. Furthermore, it is straightforward to see that for a restricted set of parity-check matrices, automorphism group decoders trade redundant rows with automorphisms. This is formally described by the following lemma, the proof of which is straightforward and hence omitted.

Lemma 5.4: Let $\mathcal{C}$ be an $[n, k, d]$ code. Then

$$
\rho_{s+1}(\mathcal{C}) \leq(n-k) \times \mathbf{S}_{s}^{\star}(\mathcal{C})
$$

for all $1 \leq s \leq d-1$, provided that a $\mathbf{s - S A D}(\mathcal{C})$ set exists.

One class of codes for which it is straightforward to prove the existence of certain SAD sets is the class of codes with transitive automorphism groups, described below.

Definition 5.5: A group $\Gamma$ of permutations of the symbols $\{0,1, \ldots, n-1\}$ is transitive if for any two symbols $i, j$ there exists a permutation $\pi \in \Gamma$ such that $i \pi=j$. A group is said to be $t$-fold transitive if for any two collections of $t$ distinct numbers $i_{1}, \ldots, i_{t} \in\{0, \ldots, n-1\}$ and $j_{1}, \ldots, j_{t} \in\{0, n-1\}$, there exists a $\pi \in \Gamma$ such that $i_{1} \pi=j_{1}, \ldots, i_{t} \pi=j_{t}$.

Lemma 5.6: Let $\mathcal{C}$ be a code with an $s$-transitive automorphism group. Then there exist b-SAD sets of $\mathcal{C}$ for all $b \leq s$.

Proof: Let $\boldsymbol{H}$ be of the form $[\boldsymbol{A} \mid \boldsymbol{I}]$. Clearly, the positions of $\boldsymbol{H}$ indexed by $k$ through $n-1$ are free of stopping sets of size $s \leq n-k$. Since the automorphism group of $\mathcal{C}$ is $s$-transitive, any collection of not more than $s$ coordinates in $\{0, \ldots, n-1\}$ is moved by some element of $\operatorname{Aut}(\mathcal{C})$ into the positions indexed by $k$ to $n-1$. Consequently, the automorphism group itself represents a (possibly non-minimal) S-SAD set.

Finding SAD sets of codes is a very complicated task, so that we focus our attention on deriving bounds on the size of such sets for specific examples of codes.

Example 5.1: Consider the $[24,12,8]$ extended Golay code $\mathcal{G}_{24}$. Since the automorphism group of the extended Golay code is 5-fold transitive, a 5-SAD set exists, and $\mathbf{S}_{5}^{\star}\left(\mathcal{G}_{24}\right) \leq\left|\mathcal{M}_{24}\right|$, where $\left|\mathcal{M}_{24}\right|$ denotes the order of the Mathieu group $\mathcal{M}_{24}$. It is well known that $\left|\mathcal{M}_{24}\right|=244823040$.

One can actually show a much stronger result, described below.

Theorem 5.7: The automorphism redundancy of the $\mathcal{G}_{24}$ code is upper bounded by 14, i.e. $\mathbf{S}_{7}^{\star}\left(\mathcal{G}_{24}\right) \leq 14$.

Proof: The proof of the result is constructive. Below, we list the particular form of $\boldsymbol{H}$ used to meet the claimed result, as well as the corresponding set of SAD automorphisms. The matrix in question is $\boldsymbol{H}\left(\mathcal{G}_{24}\right)=\left[\boldsymbol{I}_{12} \mid \boldsymbol{M}\right]$, where

$$
\boldsymbol{M}=\left[\begin{array}{cccc}
I_{3} & A & A^{2} & A^{4} \\
A & I_{3} & A^{4} & A^{2} \\
A^{2} & A^{4} & I_{3} & A \\
A^{4} & A^{2} & A & I_{3}
\end{array}\right], \quad A=\left[\begin{array}{lll}
1 & 1 & 1 \\
1 & 0 & 0 \\
1 & 0 & 1
\end{array}\right] .
$$

The automorphisms are of the form $\theta^{i} \times \psi^{j}, i=0,1, j=0,1, \ldots, 5,6$, with

$$
\begin{aligned}
& \theta=(0,12)(1,13)(2,14)(3,15) \ldots(10,22)(11,23) ; \\
& \psi=(3,6,15,9,21,18,12)(4,7,16,10,22,19,13)(5,8,17,11,23,20,14) ;
\end{aligned}
$$

Both permutations are listed in standard cycle form. Note that these automorphisms include the identity permutation for $i=j=0$. Incidentally, this is the same matrix that was studied in [25], where it was used to show that $\mathbf{P D}\left(\mathcal{G}_{24}\right)=14$.

Henceforth, the matrix obtained by combining the $\boldsymbol{H}\left(\mathcal{G}_{24}\right)$-images of all the listed automorphisms is denoted by $\boldsymbol{H}_{\mathrm{W}}$. The subscript $W$ refers to the name of the first author to have studied this matrix - Wolfmann [25].

Equation (14) allows one to bound $\rho_{6}\left(\mathcal{G}_{24}\right) \leq 12 \times 14=168$, which is significantly larger than the constructive bound provided by the matrix in Equation (16) below, which consists of 21 rows only. The matrix was constructed by identifying the $\operatorname{cog}$ of the $[23,12,7]$ code that leads to optimal stopping distance properties, generating a $m=21$ cyclic parity-check matrix, and then adding a single (fixed) parity-check position to each of the rows. 


$$
\boldsymbol{H}_{[24,12]}=\left(\begin{array}{lllllllllllllllllllllllll}
1 & 0 & 0 & 0 & 0 & 1 & 1 & 0 & 0 & 1 & 0 & 1 & 1 & 0 & 0 & 0 & 1 & 0 & 1 & 0 & 0 & 0 & 0 & 0 \\
0 & 1 & 0 & 0 & 0 & 0 & 1 & 1 & 0 & 0 & 1 & 0 & 1 & 1 & 0 & 0 & 0 & 1 & 0 & 1 & 0 & 0 & 0 & 0 \\
0 & 0 & 1 & 0 & 0 & 0 & 0 & 1 & 1 & 0 & 0 & 1 & 0 & 1 & 1 & 0 & 0 & 0 & 1 & 0 & 1 & 0 & 0 & 0 \\
0 & 0 & 0 & 1 & 0 & 0 & 0 & 0 & 1 & 1 & 0 & 0 & 1 & 0 & 1 & 1 & 0 & 0 & 0 & 1 & 0 & 1 & 0 & 0 \\
0 & 0 & 0 & 0 & 1 & 0 & 0 & 0 & 0 & 1 & 1 & 0 & 0 & 1 & 0 & 1 & 1 & 0 & 0 & 0 & 1 & 0 & 1 & 0 \\
1 & 0 & 0 & 0 & 0 & 1 & 0 & 0 & 0 & 0 & 1 & 1 & 0 & 0 & 1 & 0 & 1 & 1 & 0 & 0 & 0 & 1 & 0 & 0 \\
0 & 1 & 0 & 0 & 0 & 0 & 1 & 0 & 0 & 0 & 0 & 1 & 1 & 0 & 0 & 1 & 0 & 1 & 1 & 0 & 0 & 0 & 1 & 0 \\
1 & 0 & 1 & 0 & 0 & 0 & 0 & 1 & 0 & 0 & 0 & 0 & 1 & 1 & 0 & 0 & 1 & 0 & 1 & 1 & 0 & 0 & 0 & 0 \\
0 & 1 & 0 & 1 & 0 & 0 & 0 & 0 & 1 & 0 & 0 & 0 & 0 & 1 & 1 & 0 & 0 & 1 & 0 & 1 & 1 & 0 & 0 & 0 \\
0 & 0 & 1 & 0 & 1 & 0 & 0 & 0 & 0 & 1 & 0 & 0 & 0 & 0 & 1 & 1 & 0 & 0 & 1 & 0 & 1 & 1 & 0 & 0 \\
0 & 0 & 0 & 1 & 0 & 1 & 0 & 0 & 0 & 0 & 1 & 0 & 0 & 0 & 0 & 1 & 1 & 0 & 0 & 1 & 0 & 1 & 1 & 0 \\
1 & 0 & 0 & 0 & 1 & 0 & 1 & 0 & 0 & 0 & 0 & 1 & 0 & 0 & 0 & 0 & 1 & 1 & 0 & 0 & 1 & 0 & 1 & 0 \\
1 & 1 & 0 & 0 & 0 & 1 & 0 & 1 & 0 & 0 & 0 & 0 & 1 & 0 & 0 & 0 & 0 & 1 & 1 & 0 & 0 & 1 & 0 & 0 \\
0 & 1 & 1 & 0 & 0 & 0 & 1 & 0 & 1 & 0 & 0 & 0 & 0 & 1 & 0 & 0 & 0 & 0 & 1 & 1 & 0 & 0 & 1 & 0 \\
1 & 0 & 1 & 1 & 0 & 0 & 0 & 1 & 0 & 1 & 0 & 0 & 0 & 0 & 1 & 0 & 0 & 0 & 0 & 1 & 1 & 0 & 0 & 0 \\
0 & 1 & 0 & 1 & 1 & 0 & 0 & 0 & 1 & 0 & 1 & 0 & 0 & 0 & 0 & 1 & 0 & 0 & 0 & 0 & 1 & 1 & 0 & 0 \\
0 & 0 & 1 & 0 & 1 & 1 & 0 & 0 & 0 & 1 & 0 & 1 & 0 & 0 & 0 & 0 & 1 & 0 & 0 & 0 & 0 & 1 & 1 & 0 \\
1 & 0 & 0 & 1 & 0 & 1 & 1 & 0 & 0 & 0 & 1 & 0 & 1 & 0 & 0 & 0 & 0 & 1 & 0 & 0 & 0 & 0 & 1 & 0 \\
1 & 1 & 0 & 0 & 1 & 0 & 1 & 1 & 0 & 0 & 0 & 1 & 0 & 1 & 0 & 0 & 0 & 0 & 1 & 0 & 0 & 0 & 0 & 0 \\
0 & 1 & 1 & 0 & 0 & 1 & 0 & 1 & 1 & 0 & 0 & 0 & 1 & 0 & 1 & 0 & 0 & 0 & 0 & 1 & 0 & 0 & 0 & 0 \\
1 & 0 & 1 & 0 & 1 & 1 & 1 & 0 & 0 & 0 & 1 & 1 & 0 & 0 & 0 & 0 & 0 & 0 & 0 & 0 & 0 & 0 & 0 & 1
\end{array}\right)
$$

For the extended Golay code, one can show an even stronger result which allows for achieving decoding performance close to that of maximum likelihood (ML) decoders. This result will be discussed in detail in the next section.

In this setting, the parity-check matrix of the code $\boldsymbol{H}_{[24,12], \star}$ is of the form shown below, and the SAD set consists of a set of 23 automorphisms $\epsilon, \tau, \tau^{2}, \ldots, \tau^{22}$, where $\epsilon$ denotes the identity element of $S_{24}$ (the symmetric group of order 24), and $\tau=\left(\begin{array}{lllll}0 & 1 & 2 & \ldots & 21 \\ 22\end{array}\right)(23)$. The matrix $\boldsymbol{H}_{[24,12], \star}$ consists of a subset of distinct cogs of the extended Golay code, which allows for covering all parity-check equations in the orbits of the chosen cogs.

It can be shown that for this particular combination of parity-check matrix and SAD set, all uncorrectable erasure patterns of weight up to 11 correspond to codewords of the code (see Section VII for more details).

$$
\boldsymbol{H}_{[24,12], \star}=\left(\begin{array}{lllllllllllllllllllllllll}
1 & 1 & 1 & 0 & 0 & 0 & 0 & 0 & 1 & 0 & 0 & 1 & 1 & 0 & 0 & 0 & 0 & 0 & 1 & 0 & 0 & 0 & 0 & 1 \\
1 & 1 & 0 & 0 & 0 & 0 & 1 & 0 & 0 & 0 & 0 & 1 & 0 & 0 & 1 & 1 & 1 & 0 & 0 & 0 & 0 & 0 & 0 & 1 \\
1 & 1 & 0 & 1 & 0 & 0 & 1 & 0 & 1 & 0 & 1 & 0 & 0 & 1 & 0 & 0 & 0 & 0 & 0 & 0 & 0 & 0 & 0 & 1 \\
1 & 1 & 1 & 0 & 0 & 0 & 1 & 1 & 0 & 0 & 0 & 0 & 0 & 0 & 0 & 0 & 0 & 0 & 0 & 1 & 0 & 1 & 0 & 1 \\
1 & 1 & 0 & 0 & 0 & 1 & 0 & 0 & 0 & 1 & 0 & 1 & 0 & 1 & 0 & 0 & 0 & 0 & 0 & 0 & 0 & 1 & 0 & 1 \\
1 & 1 & 0 & 1 & 0 & 0 & 0 & 1 & 0 & 1 & 0 & 0 & 0 & 0 & 0 & 0 & 1 & 0 & 1 & 0 & 0 & 0 & 0 & 1 \\
0 & 1 & 1 & 0 & 0 & 0 & 1 & 0 & 0 & 0 & 0 & 1 & 0 & 1 & 0 & 0 & 0 & 1 & 0 & 0 & 1 & 0 & 0 & 1 \\
1 & 1 & 0 & 1 & 1 & 0 & 0 & 0 & 0 & 0 & 0 & 1 & 0 & 0 & 0 & 0 & 0 & 0 & 0 & 1 & 1 & 0 & 0 & 1 \\
1 & 1 & 1 & 1 & 0 & 1 & 0 & 0 & 0 & 0 & 0 & 0 & 0 & 0 & 1 & 0 & 0 & 1 & 0 & 0 & 0 & 0 & 0 & 1 \\
1 & 1 & 0 & 0 & 1 & 0 & 0 & 0 & 0 & 0 & 1 & 0 & 0 & 0 & 1 & 0 & 0 & 0 & 1 & 0 & 0 & 1 & 0 & 1 \\
0 & 0 & 1 & 1 & 0 & 0 & 1 & 0 & 1 & 0 & 0 & 1 & 0 & 0 & 1 & 0 & 0 & 0 & 0 & 1 & 0 & 0 & 0 & 1 \\
0 & 0 & 1 & 1 & 0 & 1 & 0 & 1 & 0 & 0 & 1 & 1 & 0 & 0 & 1 & 0 & 0 & 0 & 0 & 0 & 0 & 0 & 1 & 0
\end{array}\right)
$$

We conclude this brief overview by pointing out that there exists a strong connection between the problem of set coverings [26] and the problem of finding PD and SAD sets. This relationship can be used to develop simple heuristic search strategies for parity-check matrices that have good SAD-set properties.

For this purpose, assume that the parity-check matrix is in systematic form, with parity positions confined to the coordinates $k, \ldots, n-1$. The number of automorphisms $N_{A}$ needed to move any erasure patterns of cardinality $\leq d-1$ into the last $n-k$ positions (free of stopping sets of any size) has to satisfy

$$
N_{A} \geq\left\lfloor\frac{n}{k}\left\lfloor\frac{n-1}{k-1} \ldots\left\lfloor\left\lfloor\frac{n-d+2}{k-d+2}\right\rfloor \ldots\right\rfloor\right\rfloor,\right.
$$

which follows from a result by Schoenheim [26], who derived it in the context of set coverings.

The crux of the heuristic SAD-set search approach lies in identifying subsets of columns of $\boldsymbol{H}$ that have cardinality larger than $n-k$ and that are free of stopping sets of size up to and including $d-1$, and in combining these results with ideas borrowed from set covering theory.

For example, it is straightforward to show that the first 15 columns of the parity-check matrix in Equation (15) are free of stopping sets of size less than eight, and that any collection of more than 15 columns must contain a stopping set of size smaller than eight. Formula (17) shows that in this case, the smallest number of automorphisms required to map any set of not more than seven coordinates of a codeword into the first 15 positions is at least 60 (the same bound, for 12 positions only, equals 498). Clearly, there is no guarantee that there exist sufficiently many automorphisms that map arbitrary collections of coordinates to these positions. Nevertheless, extensive computer simulations reveal that a good strategy for identifying small SAD sets is to use a parity-check matrix in systematic form, to find a large collection of columns $K$ free of stopping sets of 
size less than $d$, and then perform a search for automorphisms that map subsets of positions in $I-K$ to positions in $K$. This method usually produces good results when the underlying codes have a large automorphism group.

\section{Automorphism Group Decoders For the BEC}

In order to distinguish between iterative decoders that use automorphisms to reduce errors due to stopping sets and standard permutation decoders, we refer to the former as automorphism group decoders. Automorphism group decoders offer one significant advantage over iterative decoders operating on redundant parity-check matrices: they have low hardware complexity (since only $n-k$ rows of the parity-check matrix are stored, along with very few permutations) and at the same time excellent decoding performance. This is, to a certain degree, offset by the slightly increased computational complexity of the decoders. The results presented in Section VII show that these additional expenses are negligible for error rates of interest.

We describe next automorphism group decoders (AGD) for cyclic codes, and also provide an example pertaining to extended cyclic codes. We restrict our attention to these classes of codes since large subgroups of the automorphism group of such codes are known and since the implementation complexity of AGD decoders in this case is very small. Nevertheless, the described decoding techniques can be applied to other classes of codes for which some information about the automorphism group is available. For the case of extended cyclic codes, it is tacitly assumed that the overall parity-check bit is confined to the last position of the codewords and that its index is $n-1$.

Throughout the section, we make use of the following result.

Theorem 6.1: ([11, Ch. 8]) Let $\boldsymbol{c}=\left(c_{0}, c_{1}, \ldots, c_{n-1}\right)$ be a codeword of an $[n, k, d]$ cyclic code. The automorphism group of the code contains the following two sets of permutations, denoted by $C_{1}$ and $C_{2}$ :

$C_{1}$ : The set of cyclic permutations $\gamma^{0}, \gamma^{1}, \ldots, \gamma^{n-1}$, where $\gamma: i \rightarrow i+1 \bmod n$;

$C_{2}$ : The set of permutations $\zeta^{0}, \zeta^{1}, \ldots, \zeta^{-1}$, where $\zeta: i \rightarrow 2 \cdot i \bmod n$, and where $c$ denotes the cardinality of the cyclotomic coset (of the $n$-th roots of unity) that contains the element one.

For extended cyclic codes, we use the same notation $C_{1}$ and $C_{2}$ to describe permutations that fix $c_{n-1}$ and act on the remaining coordinates as described in the theorem above. All automorphisms can be decomposed into products of disjoint cycles. Permutations in $C_{1}$ have one single cycle (or two cycles, for the case of extended cyclic codes), while permutations in $C_{2}$ have a number of cycles that equals the number of cyclotomic cosets $r$ of the $n$-roots of unity (or $r+1$ cycles, for the case of extended cyclic codes). The number of cycles in the automorphisms used for decoding influences the hardware complexity of the scheme and should be kept small.

$\mathrm{AGD}_{A}$ Decoders: These decoders use permutations drawn from the set $C_{1}$, which reduces the permuter architecture to one single shift register. One way to perform automorphism group decoding is to set the permuter to $\gamma^{0}$, until the presence of a stopping set is detected. In that case, the $\mathrm{AGD}_{A}$ decoder applies a randomly chosen cyclic shift $\gamma^{i}$ to the current word, $i \neq 0$. If the iterative decoder encounters another stopping set, the whole process is repeated with a (yet another) randomly chosen cyclic permutation. The decoding process terminates if either all permutations in $C_{1}$ are tested or if the decoder successfully recovers the codeword.

Assume that the number of cogs of a cyclic code is at least $n-k$. In this case, the parity-check matrix used for decoding consists of $n-k$ different cogs, provided that such a matrix has full row-rank. The redundant parity-check matrix consisting of the collection of all vectors in the $C_{1}$-orbits of the cogs is henceforth denoted by $\boldsymbol{H}_{\mathrm{AGD}_{A}}$.

$\mathrm{AGD}_{B}$ Decoders: These decoders use permutations drawn from both $C_{1}$ and $C_{2}$, so that the resulting permuter architecture is slightly more complex than that of $\mathrm{AGD}_{A}$ decoders. If a stopping set is encountered, the decoder first tries to resolve this set by applying a randomly chosen permutation from $C_{1}$. Only after the whole set $C_{1}$ is exhausted, a randomly chosen permutation from $C_{2}$ is applied to the current decoder output. The parity-check matrix used for this decoder is generated in terms of a greedy procedure. First, an arbitrary $\operatorname{cog}$ is chosen. Then, another $\operatorname{cog}$ that is not in the orbit of the first cog under any permutation in $C_{2}$ is chosen, if such a $\operatorname{cog}$ exists. The procedure is repeated until either the set of cyclic orbit generators is exhausted or until the matrix contains $n-k$ rows. In the former case, additional rows are chosen from the set of second-smallest weight codewords of the dual code, provided that they give rise to a matrix of row-rank $n-k$.

Figure 1(a) and Figure 1(b) illustrate the permutation operations used in the described decoding architectures. Here, the check nodes and edges in the graph of an extended cyclic code are fixed, while the variable nodes are permuted according to the permutations $C_{1}$ and $C_{2}$ defined in Theorem 6.1 .

The set of minimum weight $\operatorname{cogs}$ can be partitioned into $\operatorname{cog}$ families $(C F)$, where all members of one given family have identical intersection numbers. More precisely, a $C F$ is a set of cogs with identical intersection cardinalities $\left|\Sigma_{\sigma, i} \cap \Sigma_{\sigma, j}\right|$, and consequently, identical values of $S_{\sigma, 2}$, as given in Equation (13). This result is a consequence of the fact that $\operatorname{cogs}$ within the same family have the same parameters $|X Y|_{\kappa}$, introduced in Lemma 4.5

Cogs from the family with optimal intersection numbers are good candidates for constructing parity-check matrices amenable for automorphism group decoding. In this case, it is important to select subsets of cogs that are not images of each other under the automorphisms used by the decoder. For example, for the $[23,12,7]$ Golay code, the 22 cogs of minimum weight eight can be grouped into two families $C F_{1}$ and $C F_{2}$, each consisting of $11 \mathrm{cogs}$. Both families are closed under the permutations in $C_{2}$. 


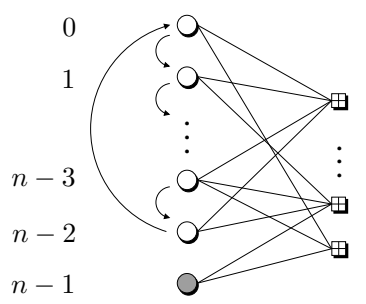

(a) $C_{1}$

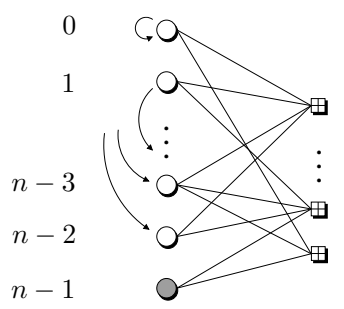

(b) $C_{2}$

Fig. 1. Permutations of $C_{1}$ and $C_{2}$ for extended cyclic codes.

TABLE VIII

INTERSECTION NUMBERS OF THE $C F_{1}$ AND $C F_{2}$ FAMILIES OF THE $[23,12,7]$ GolAy CODE.

\begin{tabular}{c|c|ccc|}
\hline$C F_{1}$ & \multicolumn{4}{|c|}{ Intersection numbers } \\
$\kappa \in$ & $\left|O O_{\kappa}\right|$ & $\left|O Z_{\kappa}\right|$ & $\left|Z O_{\kappa}\right|$ & $\left|Z Z_{\kappa}\right|$ \\
\hline$\Psi_{1},\left|\Psi_{1}\right|=2$ & 0 & 8 & 8 & 7 \\
$\Psi_{2},\left|\Psi_{2}\right|=8$ & 4 & 4 & 4 & 11 \\
$\Psi_{3},\left|\Psi_{3}\right|=12$ & 2 & 6 & 6 & 9 \\
\hline \multicolumn{5}{|c|}{ Intersection numbers } \\
\hline$C F_{2}$ & $\left|O O_{\kappa}\right|$ & $\left|O Z_{\kappa}\right|$ & $\left|Z O_{\kappa}\right|$ & $\left|Z Z_{\kappa}\right|$ \\
$\kappa \in$ & 4 & 4 & 4 & 11 \\
\hline$\Psi_{4},\left|\Psi_{4}\right|=6$ & 4 & 6 & 6 & 9 \\
$\Psi_{4},\left|\Psi_{4}\right|=16$ & 2 &
\end{tabular}

We partition the set of shift indices $\kappa, \kappa \in\{1,2, \ldots, n-1\}$, into subsets $\Psi_{i}$ which have equal parameters $\left|O O_{\kappa}\right|,\left|O Z_{\kappa}\right|$, $\left|Z O_{\kappa}\right|$, and $\left|Z Z_{\kappa}\right|$. Table VIII lists a selected subset of intersection numbers for cogs in the $C F_{1}$ and $C F_{2}$ families. It can be seen from the table that every $\operatorname{cog}$ in $C F_{1}$ has parameters $\left|O O_{\kappa}\right|=0,\left|O Z_{\kappa}\right|=\left|Z O_{\kappa}\right|=8$, and $\left|Z Z_{\kappa}\right|=7$ for the two shift indices forming $\Psi_{1}$.

For the $[31,16,7] \mathrm{BCH}$ code, the set of $15 \mathrm{cogs}$ of weight eight forms one single family $C F$. A selected set of intersection numbers of this family is shown in Table IX

\section{Results}

We present next a selected set of simulation results for the bit error rate (BER) and frame error rate (FER) performance of both redundant parity-check matrices and various automorphism group decoders. The BER equals half of the fraction of the residual erasures, as at the end of the decoding procedure one can guess all uncorrectable bits. A frame error is declared if at least one symbol in the frame estimated by the decoder does not match the corresponding symbol in the transmitted frame. Our results indicate that the FER performance of codes operating in the above described manner can be improved by adding an additional "guessing" feature, which is described in more details in the concluding part of the section.

\section{A. The Golay and Extended Golay Code}

The residual bit error rate performance of edge-removal decoding on the cyclic parity-check matrix representation based on $\operatorname{cog}_{23,12, \mathrm{~A}}$ is shown in Figure 2(a) In this figure, the cyclic $23 \times 23$ matrix is truncated to 11, 16, 18, and 23 rows, corresponding to the upper bounds on the stopping redundancy hierarchy obtained from this cyclic representation. For comparison, the performance of the ML decoder is also shown in the same figure.

In Table $\mathrm{X}$ we list the number of uncorrectable erasure patterns of size up to $\sigma=12$ in several parity-check matrices of the $[24,12,8]$ extended Golay code; $\boldsymbol{H}_{[24,12], \star}$ and $\boldsymbol{H}_{\mathrm{W}}$ were defined in Section $\nabla$ while the matrix $\boldsymbol{H}_{\mathrm{HS}}$ corresponds to the matrix of dimension $34 \times 24$ described in [4]. The index ML refers to the erasure patterns that cannot be decoded by an ML

TABLE IX

INTERSECTION NUMBERS OF THE UNIQUE $C F$ OF THE $[31,16,7]$ BCH CODE.

\begin{tabular}{|c|cccc|}
\hline$C F$ & \multicolumn{4}{|c|}{ Intersection numbers } \\
$\kappa \in$ & $\left|O O_{\kappa}\right|$ & $\left|O Z_{\kappa}\right|$ & $\left|Z O_{\kappa}\right|$ & $\left|Z Z_{\kappa}\right|$ \\
\hline$\Psi^{1},\left|\Psi^{1}\right|=2$ & 4 & 4 & 4 & 19 \\
$\Psi^{2},\left|\Psi^{2}\right|=4$ & 0 & 8 & 8 & 15 \\
$\Psi^{3},\left|\Psi^{3}\right|=24$ & 2 & 6 & 6 & 17 \\
\hline
\end{tabular}


decoder. Figures 2(b), 2(c), and 2(d) show the performance of iterative decoders operating on a selection of the parity-check matrices in Table $\mathbf{X}$ using standard BP decoding on $\boldsymbol{H}_{[24,12], \star}$ and using $\mathrm{AGD}_{A}$ and $\mathrm{AGD}_{B}$ decoders.

As can be seen, there is a significant performance gain of $\mathrm{AGD}_{A}$ or $\mathrm{AGD}_{B}$ decoders when compared to that of standard edge-removal decoders operating on the redundant parity-check matrix described in [4] or on any other non-redundant Tanner graph. In fact, the BER performance of $\mathrm{AGD}_{A}$ and $\mathrm{AGD}_{B}$ decoders approaches the performance of ML decoders. For $\mathrm{EP} \leq 0.15$, all matrix representations require an almost identical average number of iterations, indicated by the vertical bar in Figures 2(b), 2(c), and 2(d)

TABLE X

UNCORRECTABLE ERASURE PATTERNS OF THE [24, 12, 8] EXTENDED GOLAY CODE.

\begin{tabular}{|c|c|c|c|c|c|}
\hline & \multicolumn{5}{|c|}{ Number of uncorrectable erasure patterns } \\
\cline { 2 - 6 }$\sigma$ & $\boldsymbol{H}_{[24,12], \star}$ on BP & $\boldsymbol{H}_{[24,12], \star}$ on $\mathrm{AGD}_{A}$ & $\boldsymbol{H}_{\mathrm{W}}$ on BP & $\boldsymbol{H}_{\mathrm{HS}}[4]$ on BP & ML \\
\hline 3 & 7 & 0 & 0 & 0 & 0 \\
4 & 190 & 0 & 0 & 0 & 0 \\
5 & 2231 & 0 & 0 & 0 & 0 \\
6 & 15881 & 0 & 0 & 0 & 0 \\
7 & 79381 & 0 & 759 & 3284 & 759 \\
8 & 293703 & 759 & 12158 & 78218 & 12144 \\
9 & 805556 & 12144 & 93477 & 580166 & 91080 \\
10 & 1613613 & 91080 & 481764 & 1734967 & 425040 \\
11 & 2378038 & 425040 & 1547590 & 2569618 & 1313116 \\
12 & 2690112 & 1322178 & $\left(\begin{array}{c}24 \\
\sigma\end{array}\right)$ & $\left(\begin{array}{c}24 \\
\sigma\end{array}\right)$ & $\left(\begin{array}{c}24 \\
\sigma\end{array}\right)$ \\
$\geq 13$ & $\left(\begin{array}{c}24 \\
\sigma\end{array}\right)$ & $\left(\begin{array}{c}24 \\
\sigma\end{array}\right)$ & & 0 & 0 \\
\hline
\end{tabular}

\section{B. BCH Codes}

Figure 3 plots the performance of iterative edge-removal and automorphism group decoders with and without permutation features, operating on several (redundant) parity-check matrices of the $[127,113,5] \mathrm{BCH}$ code.

The standard edge-removal decoder uses parity-check matrices of cyclic form, generated by the $\operatorname{cog} \operatorname{cog}_{127,113, \mathrm{~A}}$. The matrices have $m=n-k=14$ and $m=34$ rows, respectively. The cyclic parity-check matrix with 36 rows offers significant performance improvements, when compared to its counterpart with 14 rows. Excellent BERs can also be achieved with the $\mathrm{AGD}_{A}$ decoder that operates on a parity-check matrix containing 14 rows of distinct cogs.

Figure 3 shows that the $\mathrm{AGD}_{A}$ decoder performs very close to the ML union bound, which is known to be fairly tight for the BERs considered. Again, a vertical bar (placed at $\mathrm{EP}=0.03$ ) is used to indicate the BER region for which the average number of iterations needed for successful decoding is approximately the same for all tested decoders.

Figures 4(a), 4(b), and 4(c) show the performance of several classes of iterative decoders operating on the $[31,16,7] \mathrm{BCH}$ code. The $\mathrm{AGD}_{A}$ decoder uses a parity-check matrix that consists of all 15 cogs of the code, and for comparison, standard iterative decoding is performed on the same matrix. In addition, the performance of standard iterative decoders on three different cyclic parity-check matrices with $m=n-k=15, m=21$, and $m=31$ rows is plotted as well. The generating cog of these matrices is $\operatorname{cog}_{31,16, \mathrm{~A}}$.

Performance results for the $\mathrm{BCH}$ code under consideration are presented in Figure 4(d), pertaining to the cyclic parity-check matrices based on $\operatorname{cog}_{31,16, \mathrm{~A}}$ and $\operatorname{cog}_{31,16, \mathrm{C}}$ in Table VII In all simulations, the number of rows in the parity-check matrix equals the smallest listed value in the table. By considering only the representation indexed by $\operatorname{cog}_{31,16, \mathrm{~A}}$, it can be observed that the most significant BER improvement is achieved when the matrix has $m=18$, rather than $m=15$ rows. The results also show that for short block codes, like the ones considered in this example, almost all cogs give rise to similar residual bit error rates. Nevertheless, for codes of longer length and larger co-dimension, the particular choice of the cog may have a significant bearing on the stopping set characteristics.

In all the examples investigated, the improved performance of automorphism group decoders comes at the cost of increased decoding complexity, when compared to edge-removal techniques. Clearly, to correct more erasures than standard iterative decoders, automorphism group decoders have to go through additional decoding iterations, and in addition, perform a certain number of permutation operations. A measure of computational complexity of automorphism group decoders is plotted in Figure 5. Here, the number of excess iterations (when compared to edge-removal decoding operating on the same channel output) is plotted versus the ER of the BEC. As one can see, at most four additional iterations suffice to resolve the decoding ambiguities of most error patterns that are uncorrectable by belief propagation techniques.

The results in Figures 4(a) and 4(b) show that there exists a significant gap between the performance of automorphism group decoders and ML decoders when considering FERs, rather than BERs.

To mitigate this problem, we propose to include an additional feature into the edge-removal and automorphism group decoding algorithms. The feature in question is guessing: when the decoder fails to correct a given erasure, it continues decoding by guessing the value of one of the erased bits. The idea of incorporating guessing methods into belief propagation algorithms was 


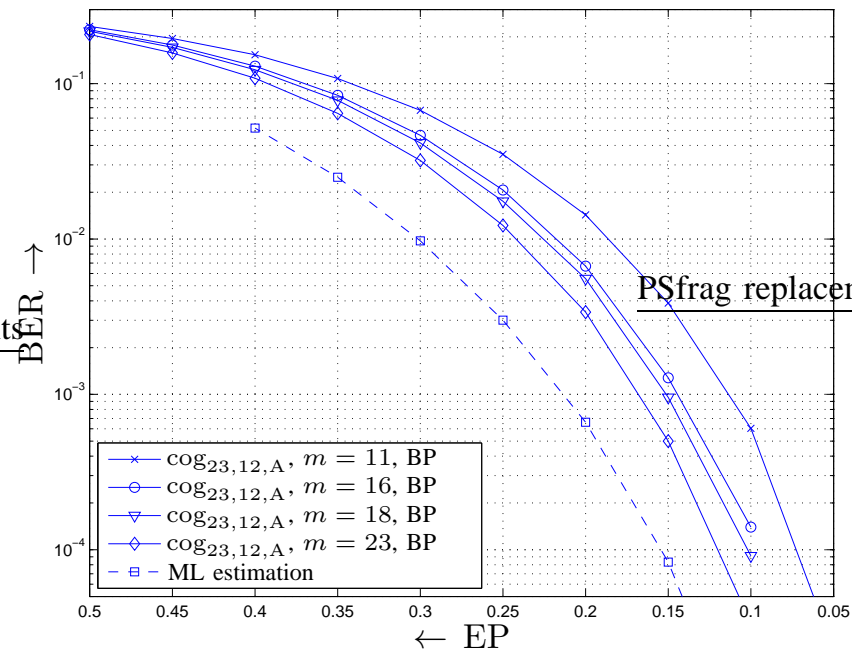

(a)

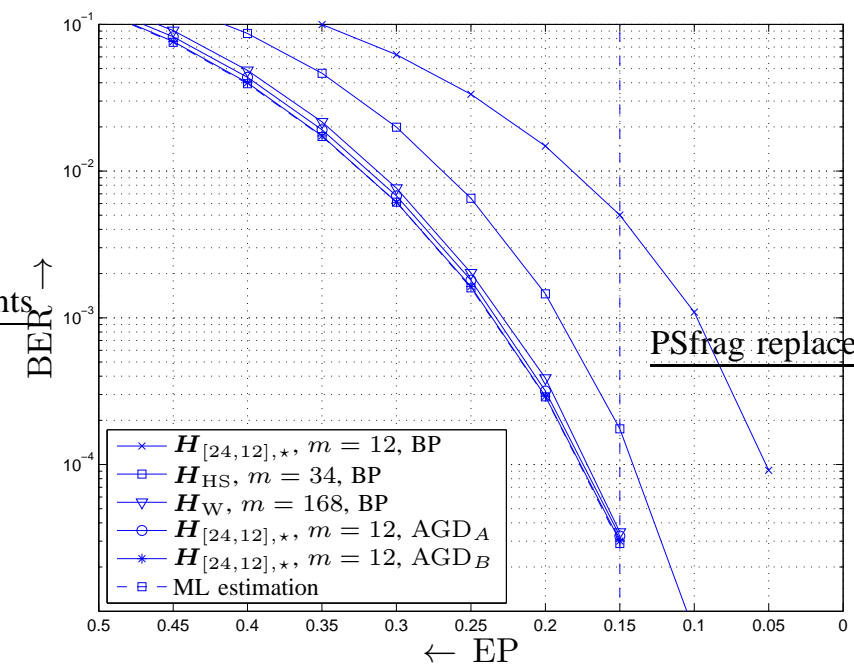

(b)

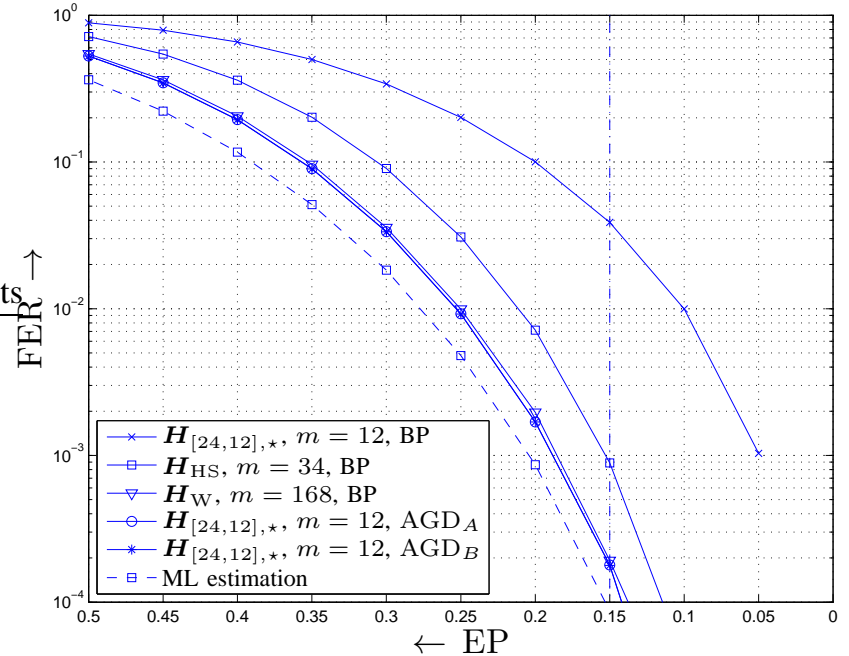

(c)

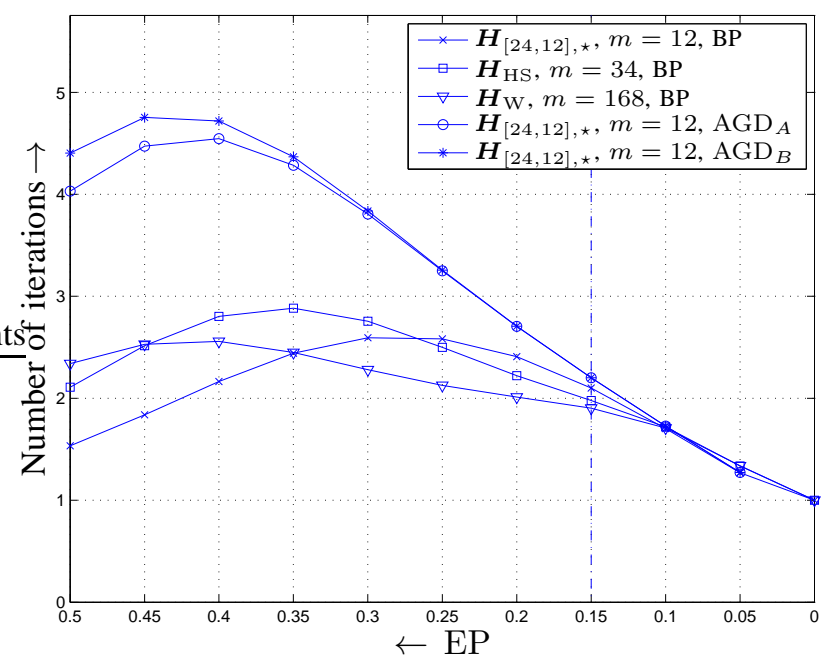

(d)

Fig. 2. Performance of the $[23,11,7]$ and $[24,12,8]$ (extended) Golay codes.
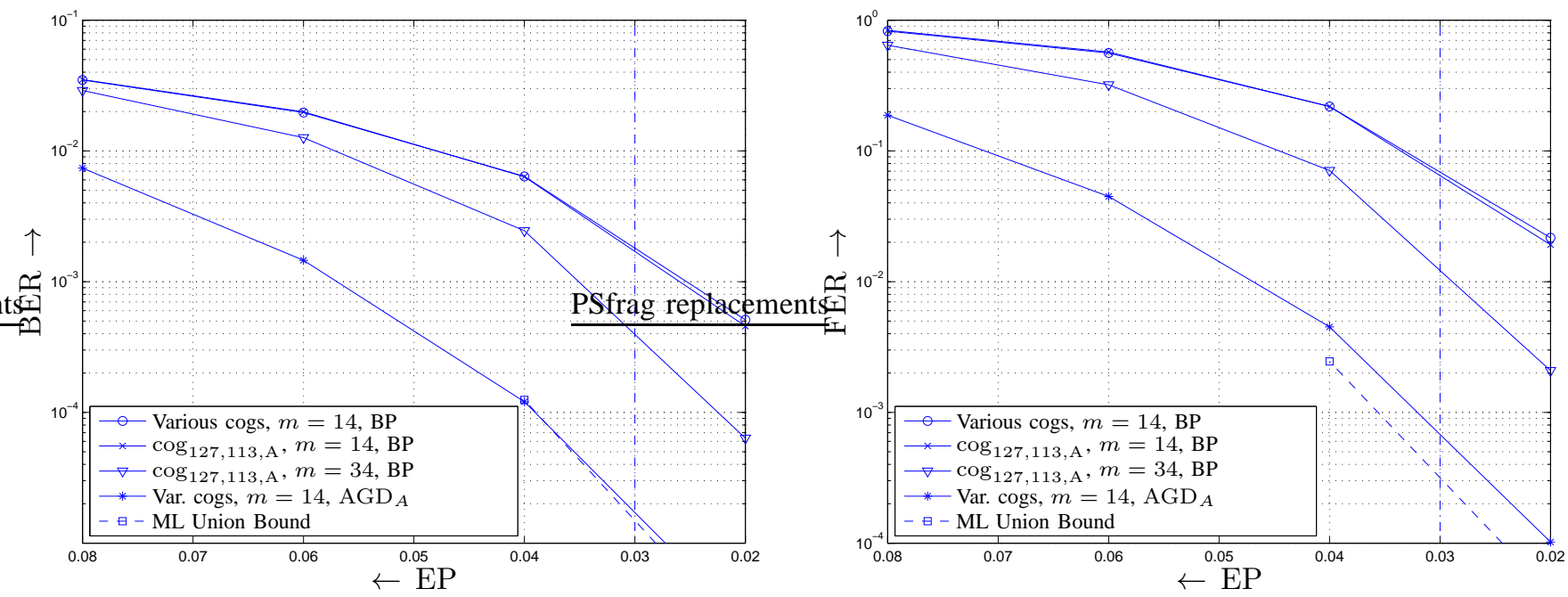

Fig. 3. Performance of the $[127,113,5]-\mathrm{BCH}$ code.

first described in [27] and, in the context of stopping set analysis in unpublished work by Han and Siegel [28]. Figure 6 shows the FER performance improvement for the $[31,16,7] \mathrm{BCH}$ code achieved through combined automorphism group decoding 


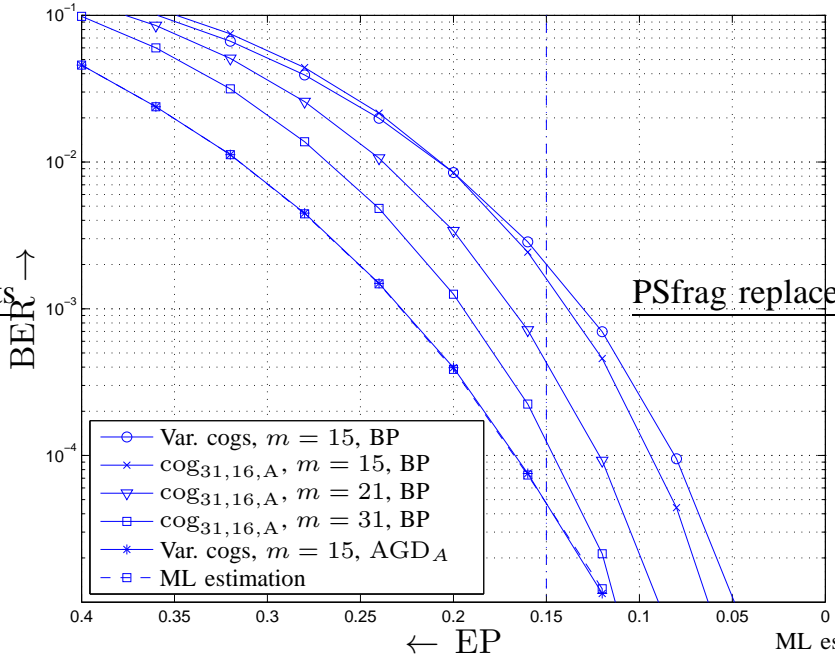

(a)

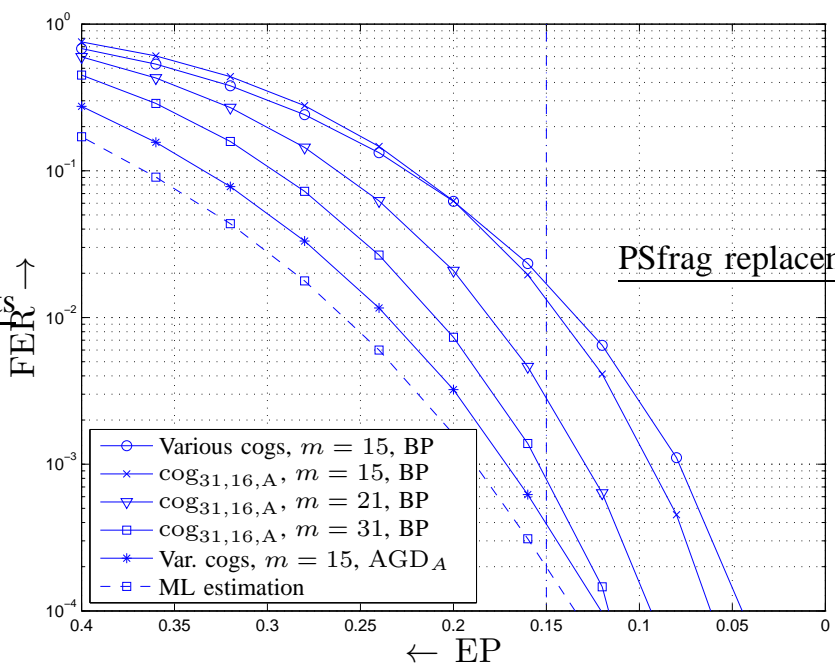

(b)

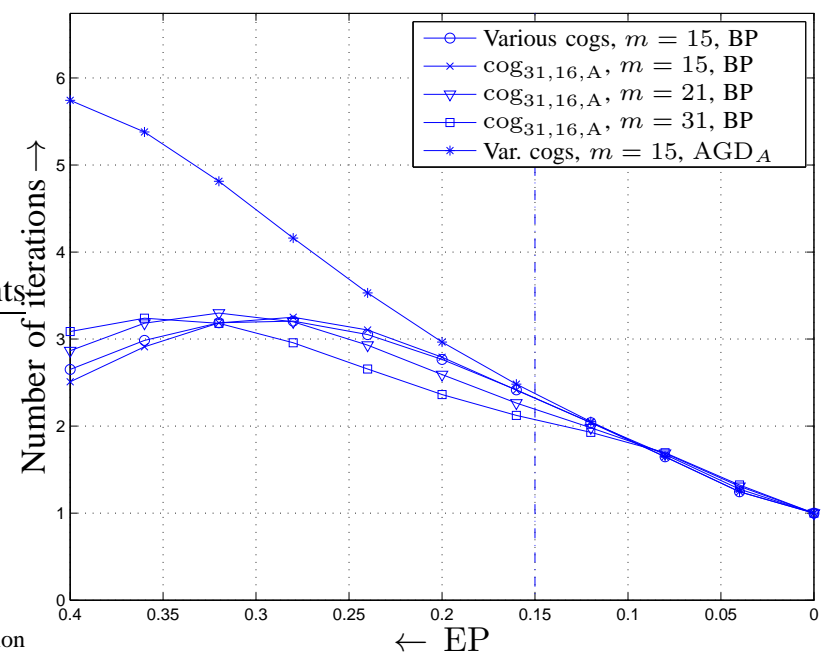

(c)

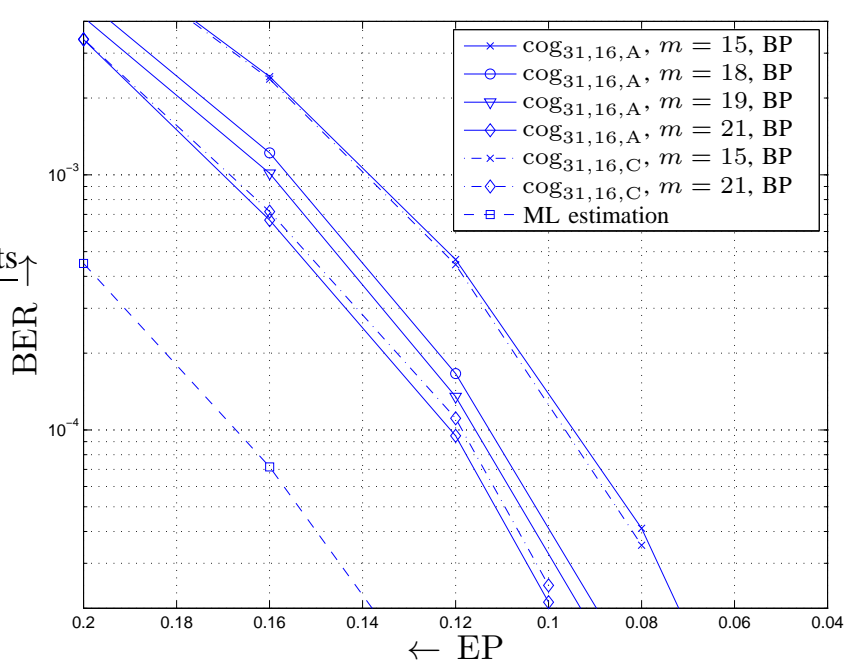

(d)

Fig. 4. Performance of the $[31,16,7]-\mathrm{BCH}$ code.

and bit guessing. As it can be observed, the FER performance of $\mathrm{AGD}_{A}$ decoders with the guessing feature approaches the performance of ML decoding.

\section{CONCLUSIONS}

We introduced the notion of the stopping redundancy hierarchy of linear block codes, and derived lower and upper bounds on the elements of this ordered list. We also investigated the stopping redundancy of cyclic-parity check matrices, and proposed new redundant parity-check matrix constructions and automorphism group decoding techniques. In this setting, we introduced the notion of a stopping set automorphism group decoding set, and related this new code invariant to the stopping redundancy hierarchy.

\section{APPENDIX I}

\section{PRoOF OF THEOREM 3.8}

We start the proof by introducing the notion of an orthogonal array [7].

Definition I.1: ([29, p.5]) An orthogonal array of strength $t$ is a matrix of $m \times n$ elements with the property that every $m \times t$ subarray contains each possible $t$-tuple the same number of times. The codewords of an $[n, k, d]$ code $\mathcal{C}$ form an orthogonal array of dimension $2^{k} \times n$ and strength $d^{\perp}-1$, where, as before, $d^{\perp}$ denotes the dual distance of $\mathcal{C}$.

It can be shown that the set of all codewords of a code forms an orthogonal array of strength $d^{\perp}-1$ [29].

Definition I.2: Let $\mathcal{C}$ be an $[n, k, d]$ code and $\mathcal{C}^{\perp}$ its dual code. The ensemble $\mathcal{M}_{\mathcal{C}}(m)$ is defined as the set of all $m \times n$ matrices with rows chosen uniformly, independently, and with replacement, from the set of $2^{n-k}$ codewords of $\mathcal{C}^{\perp}$. 


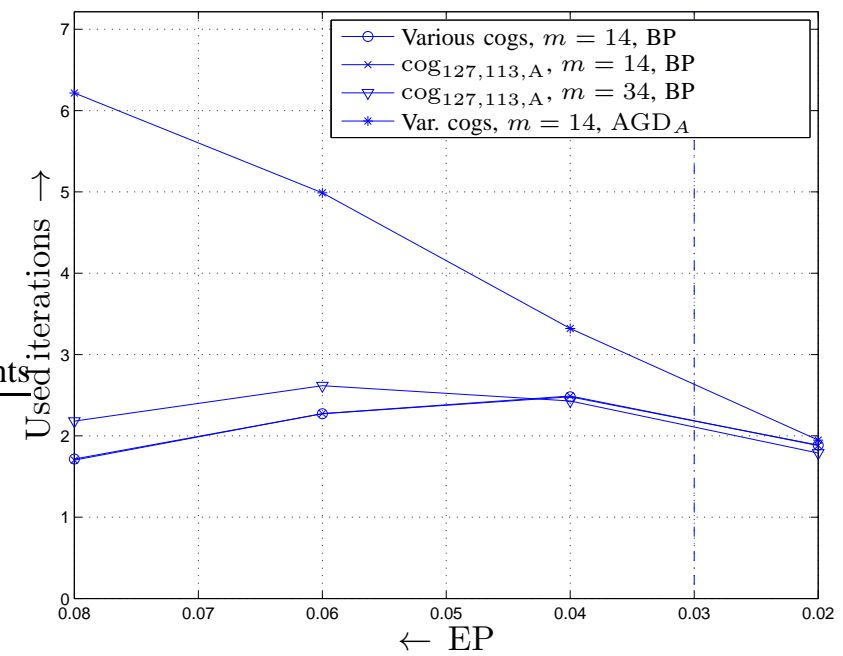

Fig. 5. Number of iterations of the automorphism group decoder for the $[127,113,5] \mathrm{BCH}$ code.

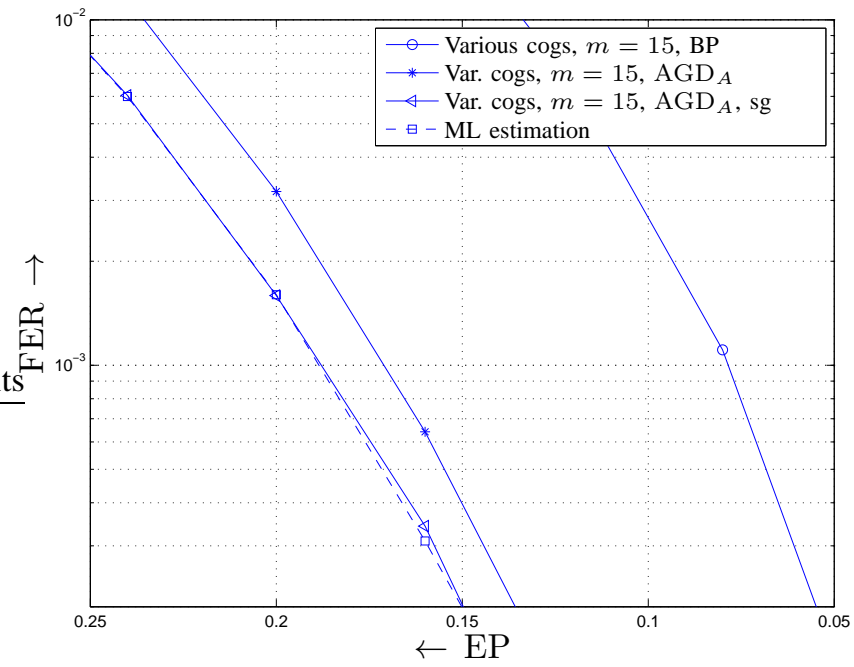

Fig. 6. Performance improvements achievable by the guessing method.

Let $\mathcal{A}$ be the set of all binary $t$-tuples of weight one. Let $E_{i}$ be the event that the $i$-th collection of $t$ columns in an array randomly drawn from the $\mathcal{M}_{\mathcal{C}}(m)$ ensemble contains no element from $\mathcal{A}$, i.e., that the $i$-th collection of columns corresponds to a stopping set of size $t$. If $\{S(t)=0\}$ denotes the event that a given array does not contain stopping sets of size $t$, then $P\left\{\cap \bar{E}_{i}\right\}=P\{S(t)=0\}$. Observe next that

$$
\begin{aligned}
P\left\{E_{i}\right\} & =P\left\{\bigcap_{k=1}^{m}\left\{k^{\text {th }} \text { row of the } i^{\text {th }} \text { restriction } \notin \mathcal{A}\right\}\right\} \\
& =\left(P\left\{1^{\text {st }} \text { row of the } i^{\text {th }} \text { restriction } \notin \mathcal{A}\right\}\right)^{m} \\
& =\left(1-t \cdot 2^{-t}\right)^{m},
\end{aligned}
$$

where the last step follows from the fact that the occurrence of every $t$-tuple in the orthogonal array is equally likely, with probability $2^{-t}$. The dependence number $\tau$ of the events $E_{i}$, defined in Equation (3.7), equals

$$
(\tau+1)=\left(\begin{array}{l}
n \\
t
\end{array}\right)-\left(\begin{array}{c}
n-t \\
t
\end{array}\right) .
$$

The above result is a consequence of the fact that two collections of $t$ columns are co-dependent if and only if they share at least one column. If one collection of $t$ columns is fixed, another collection is independent of it if its columns are chosen from the remaining $n-t$ columns. 
By inserting the expressions (I.18) and (I.19) into the defining inequality (7) of LLL gives the following sufficient condition for the existence of a parity-check matrix with $m$ rows, free of stopping sets of size $t$ :

$$
\mathrm{e}\left(1-t \cdot 2^{-t}\right)^{m}\left(\left(\begin{array}{c}
n \\
t
\end{array}\right)-\left(\begin{array}{c}
n-t \\
t
\end{array}\right)\right) \leq 1
$$

This implies that, if $m$ satisfies the condition above, there exists at least one parity-check matrix of dimension $m$ free of stopping sets of size $t$. Consequently, this bound on $m$ represents an upper bound on $\rho_{\ell}(\mathcal{C})$. is

A sufficient condition for the existence of a matrix with $m$ rows that does not contain stopping sets of size $j \in\{1,2, \ldots, t\}$,

$$
\sum_{j=1}^{t} \mathrm{e}\left(1-j \cdot 2^{-j}\right)^{m}\left(\left(\begin{array}{c}
n \\
j
\end{array}\right)-\left(\begin{array}{c}
n-j \\
j
\end{array}\right)\right) \leq 1 .
$$

By observing that

$$
\max _{1 \leq j \leq t}\left(1-j \cdot 2^{-j}\right)^{m}=\left(1-t \cdot 2^{-t}\right)^{m}
$$

we arrive at

$$
\text { e }\left(1-t \cdot 2^{-t}\right)^{m} \sum_{j=1}^{t}\left(\left(\begin{array}{c}
n \\
j
\end{array}\right)-\left(\begin{array}{c}
n-j \\
j
\end{array}\right)\right) \leq 1 .
$$

Note that Equation (I.20) can be rewritten as

$$
m \geq \frac{1+\log \sum_{j=1}^{t}\left(\left(\begin{array}{c}
n \\
j
\end{array}\right)-\left(\begin{array}{c}
n-j \\
j
\end{array}\right)\right)}{-\log \left(1-\frac{t}{2^{t}}\right)} .
$$

In order to ensure that the chosen matrix has full row-rank, it may be necessary to add $n-k-\ell+1$ additional rows.

Observe that any collection of at most $d-1$ columns of a parity-check matrix is linearly independent. This follows from the fact that the minimum distance of the code is $d$. As a consequence, in order for two collections of $t$ columns to be independent, one must have $2 \cdot t<d$. This restricts the validity of the above bound to stopping distances $\ell \leq\left\lfloor\frac{d+1}{2}\right\rfloor$ only. Setting $t=\ell-1$ completes the proof.

\section{APPENDIX II}

ASYMPTOTIC BEHAVIOR OF THE BOUND IN THEOREM 3.8

We investigate the asymptotic behavior of the bound in Theorem 3.8. Let $m^{\prime}$ be the first summand of Eq. (9), i.e.

$$
m^{\prime}=\frac{1+\log \sum_{j=1}^{\ell-1}\left(\left(\begin{array}{c}
n \\
j
\end{array}\right)-\left(\begin{array}{c}
n-j \\
j
\end{array}\right)\right)}{-\log \left(1-\frac{\ell-1}{2^{\ell-1}}\right)} .
$$

Let us consider the case $\ell-1=\lambda n$, for $0<\lambda<1$. In this case, the logarithm in the numerator grows at most linearly with $n$, since

$$
\sum_{j=1}^{\ell-1}\left(\begin{array}{l}
n \\
j
\end{array}\right)<2^{n}
$$

and the second sum under the logarithm never exceeds the first.

As a result, the asymptotic behavior of the given upper bound is dominated by the expression in the denominator, which, for large $n$, takes the form

$$
-\log \left(1-\frac{\ell-1}{2^{\ell-1}}\right) \simeq \frac{\lambda n}{2^{\lambda n}}
$$

and therefore

$$
m^{\prime} \simeq \frac{(1+n) 2^{\lambda n}}{\lambda n} \simeq \frac{2^{\lambda n}}{\lambda}
$$

Consequently, for codes with minimum distance $d=$ const. $\cdot n$, the upper bound on $m$ is exponential in $n$. 
APPENDIX III

PROOF OF THEOREM 3.9

Theorem 3.9 can be proved using arguments similar to those described in the proof of Theorem 3.8. First, observe that the number of distinct collections of columns, and consequently, the number of events $E_{i}$ used in LLL equals

$$
N=\sum_{j=1}^{t}\left(\begin{array}{l}
n \\
j
\end{array}\right)
$$

Since the dependency number for the high probability variation of Lovász Local Lemma is the same as the one given in Theorem 3.8 it follows that

$$
\left(1-t \cdot 2^{-t}\right)^{m} \leq \frac{\epsilon}{\sum_{j=1}^{t}\left(\begin{array}{c}
n \\
j
\end{array}\right)}\left(1-\frac{\epsilon}{\sum_{j=1}^{t}\left(\begin{array}{c}
n \\
j
\end{array}\right)}\right)^{\sum_{j=1}^{t}\left[\left(\begin{array}{c}
n \\
j
\end{array}\right)-\left(\begin{array}{c}
n-j \\
j
\end{array}\right)-1\right]} .
$$

After some simple algebraic manipulation, one can show that the above expression gives rise to the following bound:

$$
m \geq \frac{\log \frac{\epsilon}{\sum_{j=1}^{t}\left(\begin{array}{c}
n \\
j
\end{array}\right)}+\left(\sum_{j=1}^{t}\left[\left(\begin{array}{c}
n \\
j
\end{array}\right)-\left(\begin{array}{c}
n-j \\
j
\end{array}\right)-1\right]\right) \cdot \log \left(1-\frac{\epsilon}{\sum_{j=1}^{t}\left(\begin{array}{l}
n \\
j
\end{array}\right)}\right)}{\log \left(1-\frac{t}{2^{t}}\right)} .
$$

As a result, for a given set of code parameters, with probability greater than $1-\epsilon$ every parity-check matrix with $m$ rows has stopping distance at least $t+1$. Substituting $t=\ell-1$ completes the proof.

\section{APPENDIX IV}

\section{CYClic PARITY-CHECK MATRICES OF QR CODES}

Let $\mathcal{Q}$ be a $\mathrm{QR}$ code of prime length $n=3(\bmod 4)$, and let $N$ and $Q$ denote the set of quadratic non-residues and residues of the underlying finite field, respectively. The idempotent of the code is the polynomial $\sum_{i \leq n} f_{i} x^{i}$, where $f_{i}=1$ if $i \in N \cup\{0\}$, and is zero otherwise. The idempotent can be used as the first row of a (redundant) cyclic parity-check matrix of $\mathcal{Q}$.

In what follows, we assume for two fixed indices $i$ and $j$ that $\kappa=i-j(\bmod n)$. For a prime $n=4 t-1,|Q|=2 t-1$, and for each integer $\kappa \in Q, \kappa \neq 0(\bmod n)$, there exist exactly $t-1$ ordered pairs $(i, j), i \neq j$, such that $i, j \in Q$. Similarly, for fixed $\kappa$ and $i \in Q$, there exist $t-1$ distinct values $j \in N$ that result in the given value of $\kappa$.

The lower bound of Example 4.1 can be derived as follows. First, observe that the number of overlapping zeros between the first row and its $\kappa$-th cyclic shift, $\left|Z Z_{\kappa}\right|$, equals

$$
\left|Z Z_{\kappa}\right|=\sum_{i=0}^{n-1}\left(1-f_{i}\right)\left(1-f_{i-\kappa}\right)
$$

Let us now determine the number of pairs $(i, j), i, j \in Q$, for which $\kappa \in N$. As already pointed out, for each $\kappa \in Q$, there are $t-1$ choices for pairs $i, j \in Q$, and $t-1$ choices for $i \in Q, j \in N$. The $(2 t-1)^{2}$ pairs $i, j \in Q$ arise from $(2 t-1)(t-1)$ different values of $\kappa$, and there are $(2 t-1)$ choices for $\kappa=0$ that lead to $i \in Q$. In addition, $(2 t-1)(t-1)$ values of $\kappa$ result in $i, j \in Q, \kappa \in N$, while for $t-1$ values of $\kappa \in N$ one has $i, j \in Q$. Therefore, the number of pairs $(i, j)$ such that $i, j \in Q$ equals $t-1$ for $\kappa \in Q$, as well as for $\kappa \in N$. As there are $t-1$ quadratic residues, for $\kappa \in Q$ as well as for $\kappa \in N$, it follows that $\left|Z Z_{\kappa}\right|=t-1$ (excluding $\kappa=0$ ).

The row-weight of the cyclic parity-check matrix equals $\omega=|N|+1=2 t$, where $\omega=\left|O O_{\kappa}\right|+\left|O Z_{\kappa}\right|=\left|O O_{\kappa}\right|+\left|Z O_{\kappa}\right|$. Therefore, $\left|O Z_{\kappa}\right|=\left|Z O_{\kappa}\right|$. Furthermore, one has $\left|O O_{\kappa}\right|+\left|O Z_{\kappa}\right|+\left|Z O_{\kappa}\right|+\left|Z Z_{\kappa}\right|=n$, and the same result can be deduced from the fact that

$$
\left|O Z_{\kappa}\right|=\sum_{i=0}^{n-1} f_{i}\left(1-f_{i-\kappa}\right)=\sum_{i=0}^{n-1} f_{i}-\sum_{i=0}^{p-1} f_{i} f_{i-\kappa}=2 t-\left|O O_{\kappa}\right| .
$$

One can now derive an expression for $\left|O O_{\kappa}\right|$ as follows:

$$
\begin{aligned}
\left|O O_{\kappa}\right| & =n-\left|Z Z_{\kappa}\right|-\left|O Z_{\kappa}\right|-\left|Z O_{\kappa}\right|=n-(t-1)-2 \cdot\left|O Z_{\kappa}\right|=n-t+1-2 \cdot\left(2 t-\left|O O_{\kappa}\right|\right) \\
& =n-t+1-4 t+2 \cdot\left|O O_{\kappa}\right|, \text { i.e. } \\
\left|O O_{\kappa}\right| & =5 t-1-n=5 t-1-(4 t-1)=t,
\end{aligned}
$$

so that

$$
\left|O Z_{\kappa}\right|=\left|Z O_{\kappa}\right|=2 t-\left|O O_{\kappa}\right|=2 t-(t-2)=t
$$


Since the intersection numbers do not depend on the value of $\kappa$ and on this number being a quadratic residue or non-residue, we henceforth omit the subscript $\kappa$. Also, note that we do not make use of the intersection numbers for which $\kappa=0$, since we are interested in counting the number of stopping sets resolved by distinct rows of a (redundant) parity-check matrix.

Inserting the expressions above into the formula of Lemma 4.5 shows

$$
\left|\Sigma_{\sigma, \mu} \cap \Sigma_{\sigma, \mu+\kappa}\right|=|O O| \cdot\left(\begin{array}{c}
|Z Z| \\
\sigma-1
\end{array}\right)+|O Z| \cdot|Z O| \cdot\left(\begin{array}{c}
|Z Z| \\
\sigma-2
\end{array}\right)=\frac{n+1}{4} \cdot\left(\begin{array}{c}
\frac{n-3}{4} \\
\sigma-1
\end{array}\right)+\left(\frac{n+1}{4}\right)^{2}\left(\begin{array}{c}
\frac{n-3}{4} \\
\sigma-2
\end{array}\right),
$$

so that

$$
S_{\sigma, 2}=\sum_{\mu=1}^{m-1} \sum_{\kappa=1}^{m-\mu}\left|\Sigma_{\sigma, \mu} \cap \Sigma_{\sigma, \mu+\kappa}\right|=\left(\begin{array}{c}
m \\
2
\end{array}\right) \cdot \frac{n+1}{4} \cdot\left(\left(\begin{array}{c}
\frac{n-3}{4} \\
\sigma-1
\end{array}\right)+\frac{n+1}{4}\left(\begin{array}{c}
\frac{n-3}{4} \\
\sigma-2
\end{array}\right)\right) .
$$

It is also straightforward to show that

$$
\begin{gathered}
m\left|\Sigma_{\sigma, 1}\right|-\frac{2}{m} \cdot \sum_{\kappa=1}^{m-1}(m-\kappa)\left|\Sigma_{\sigma, 1} \cap \Sigma_{\sigma,\left((1+\kappa) \bmod ^{*} m\right)}\right|= \\
m \cdot \frac{n+1}{2} \cdot\left(\begin{array}{c}
\frac{n-1}{2} \\
\sigma-1
\end{array}\right)-(m-1) \cdot \frac{n+1}{4} \cdot\left(\left(\begin{array}{c}
\frac{n-3}{4} \\
\sigma-1
\end{array}\right)+\frac{(n+1)}{4}\left(\begin{array}{c}
\frac{n-3}{4} \\
\sigma-2
\end{array}\right)\right) .
\end{gathered}
$$

From the condition $\left|\bigcup_{i=1}^{m} \Sigma_{\sigma, i}\right| \geq\left(\begin{array}{l}n \\ \sigma\end{array}\right)$ and $\sigma=\ell-1$, we obtain a lower bound for the number of rows $\mu_{\ell}$ that a parity-check matrix consisting of cyclic shifts of the idempotent must have in order to have stopping distance at least $\ell$ :

$$
\mu_{\ell} \geq \max _{\sigma<\ell}\left\lceil\frac{\left(\begin{array}{l}
n \\
\sigma
\end{array}\right)-M}{\frac{n+1}{2}\left(\frac{n-1}{\sigma-1}\right)-M}\right\rceil .
$$

In the above equation, we used

$$
M=\frac{n+1}{4} \cdot\left(\left(\begin{array}{c}
\frac{n-3}{4} \\
\sigma-1
\end{array}\right)+\frac{(n+1)}{4}\left(\begin{array}{c}
\frac{n-3}{4} \\
\sigma-2
\end{array}\right)\right) .
$$

Note that the set of quadratic residues also forms a cyclic difference set with parameters $(n, k, \lambda)=\left(n, \frac{n-1}{2}, \frac{n-3}{4}\right)$ [30], so that the results of Section IV can be used in this context as well.

It is also of interest to find an upper bound on the stopping distance $\ell$ of a redundant $n \times n$ parity-check matrix that consists of all cyclic shifts of the idempotent of a QR code. For this purpose, we use Equation (IV.23) with $m=n$, and ask for the largest value of $\sigma$ for which

$$
\left(\begin{array}{l}
n \\
l
\end{array}\right) \leq n \cdot \frac{n+1}{2} \cdot\left(\begin{array}{c}
\frac{n-1}{2} \\
l-1
\end{array}\right)-(n-1) \cdot \frac{n+1}{4} \cdot\left(\left(\begin{array}{c}
\frac{n-3}{4} \\
l-1
\end{array}\right)+\frac{(n+1)}{4}\left(\begin{array}{c}
\frac{n-3}{4} \\
l-2
\end{array}\right)\right), 1 \leq l \leq \sigma .
$$

We point out that although the value $\sigma+1$ serves as an upper bound on the stopping distance of the parity-check matrix under consideration, there is no guarantee that the matrix itself has a stopping distance that meets this bound.

To this end, we only seek a solution for $l=\sigma$, since it can be shown that this value of the parameter imposes the tightest restriction on the inequality.

Also, for both $n$ and $\sigma$ sufficiently large (where $\sigma=o(n)$ ), we can approximate the above inequality by

$$
\left(\begin{array}{l}
n \\
\sigma
\end{array}\right) \lesssim n \sigma\left(\begin{array}{c}
n / 2 \\
\sigma
\end{array}\right)-n \sigma^{2}\left(\begin{array}{c}
n / 4 \\
\sigma
\end{array}\right)
$$

since

$$
\frac{n^{2}}{2}\left(\begin{array}{c}
n / 2 \\
\sigma-1
\end{array}\right) \simeq n \sigma\left(\begin{array}{c}
n / 2 \\
\sigma
\end{array}\right)
$$

and

$$
\frac{n^{2}}{4}\left(\left(\begin{array}{c}
n / 4 \\
\sigma-1
\end{array}\right)+\frac{n}{4}\left(\begin{array}{c}
n / 4 \\
\sigma-2
\end{array}\right)\right) \simeq n \sigma^{2}\left(\begin{array}{c}
n / 4 \\
\sigma
\end{array}\right)
$$

As the family of QR codes contains an infinite number of codes, we provide next an asymptotic analysis that allows us to upper bound the achievable stopping distance of redundant parity-check matrices based on the idempotent of the code.

To find an asymptotic lower bound for the stopping redundancy of cyclic parity-check matrices, we use the bound [11, p. 309, Eq. 16]

$$
f_{1}(\beta, n) \cdot \mathrm{e}^{n \cdot H(\beta)} \leq\left(\begin{array}{c}
n \\
\beta n
\end{array}\right) \leq f_{2}(\beta, n) \mathrm{e}^{n \cdot H(\beta)},
$$


where $\beta n$ is an integer, $H(\beta)=-\beta \ln \beta-(1-\beta) \ln (1-\beta)$ stands for Shannon's entropy in natural units, $f_{1}(\beta, n)=\frac{1}{\sqrt{8 n \beta(1-\beta)}}$, and $f_{2}(\beta, n)=\frac{1}{\sqrt{2 \pi n \beta(1-\beta)}}$.

For $\beta \rightarrow 0$, the following asymptotic formula holds for $H(\beta)$ :

$$
\begin{aligned}
H(\beta) & =-\beta \ln \beta-(1-\beta) \ln (1-\beta) \\
& =-\beta \ln \beta-\beta+\beta^{2} / 2+O\left(\beta^{3}\right) .
\end{aligned}
$$

In the same asymptotic domain, $f_{1}(\beta, n)$ and $f_{2}(\beta, n)$ take the form

$$
f_{1}(\beta, n)=\frac{1}{\sqrt{8 n \beta(1-\beta)}}=\frac{1}{\sqrt{8 n \beta}}(1+O(\beta))
$$

and

$$
f_{2}(\beta, n)=\frac{1}{\sqrt{2 \pi n \beta(1-\beta)}}=\frac{1}{\sqrt{2 \pi n \beta}}(1+O(\beta)) .
$$

Denote the lower bound for the left hand side of Equation IV.24 by $L(n, \sigma)$. Similarly, let the upper bound for the two terms on the right hand side of the same equation be denoted by $R_{1}(n, \sigma)$ and $R_{2}(n, \sigma)$, respectively.

Invoking the asymptotic expressions described above shows that

$$
\left(\begin{array}{l}
n \\
\sigma
\end{array}\right) \gtrsim \frac{n^{\sigma}}{\sqrt{8 \sigma^{2 \sigma+1}}} \mathrm{e}^{-\sigma+\sigma^{2} /(2 n)+O\left(\sigma^{3} / n^{2}\right)}(1+O(\sigma / n))=L(n, \sigma),
$$

and that

$$
\begin{gathered}
n \sigma\left(\begin{array}{c}
n / 2 \\
\sigma
\end{array}\right) \lesssim n^{2 \sigma+1} \sigma \frac{1}{\sqrt{4 \pi 2^{4 \sigma} \sigma^{4 \sigma+1}}} \mathrm{e}^{-2 \sigma+2 \sigma^{2} / n+O\left(\sigma^{3} / n^{2}\right)}(1+O(\sigma / n))=R_{1}(n, \sigma), \\
-n \sigma^{2}\left(\begin{array}{c}
n / 4 \\
\sigma
\end{array}\right) \lesssim-n^{4 \sigma+1} \sigma^{2} \frac{1}{\sqrt{324^{8 \sigma} \sigma^{8 \sigma+1}}} \mathrm{e}^{-4 \sigma+8 \sigma^{2} / n+O\left(\sigma^{3} / n^{2}\right)}(1+O(\sigma / n))=R_{2}(n, \sigma) .
\end{gathered}
$$

Tedious, but straightforward algebraic manipulation reveals that the above inequalities imply

$$
1 \lesssim \frac{\sqrt{2} \sigma n^{\sigma+1}}{\sqrt{\pi 2^{4 \sigma} \sigma^{2 \sigma}}} \mathrm{e}^{\left(-\sigma+3 \sigma^{2} / 2 n\right)}-\frac{n^{3 \sigma+1} \sigma^{2}}{2 \sqrt{4^{8 \sigma} \sigma^{6 \sigma}}} \mathrm{e}^{\left(-3 \sigma+15 \sigma^{2} / 2 n\right)}
$$

The square-root bound for the minimum distance of QR codes asserts that their minimum distance is lower bounded by $\sqrt{n}$. Although the minimum distance of many quadratic residue codes exceeds this bound, little is known about the asymptotic behavior of the exact minimum distance. We henceforth define the designed minimum distance, which equals $\sqrt{n}$, and proceed to evaluate the right hand side of the expression in the above equation for $\sigma=\sqrt{n}$. In this case, the resulting formula equals

$$
c_{1} n^{\sqrt{n}+3 / 2} \mathrm{e}^{-\sqrt{n}-2 \sqrt{n} \ln 2}-c_{2} n^{3 \sqrt{n}+2} \mathrm{e}^{-3 \sqrt{n}-4 \sqrt{n} \ln 4},
$$

for some positive constants $c_{1}$ and $c_{2}$. For $n \rightarrow \infty$, this expression diverges to $-\infty$. As a consequence, the stopping distance of redundant cyclic parity-check matrices generated by the idempotent of a QR code cannot meet the square-root bound. Furthermore, no positive fraction of this bound is attainable by a matrix of this form.

This finding implies that, rather than using idempotents as generators, cogs should be used instead, since they have significantly lower density.

\section{APPENDIX V \\ Construction of Redundant Parity-Check Matrices: Double-Error Correcting BCH Codes}

We describe the generalized Hollmann-Tolhuizen (HT) method for generating redundant parity-check matrices of doubleerror correcting BCH codes. The procedure consists of four steps. In the first step, the algorithm outlined in [10] is used to create a redundant parity-check matrix for the Hamming supercode. This matrix is used as the first sub-block in the parity-check matrix of the $\mathrm{BCH}$ code. In the second step, a second sub-block of rows is added, consisting of codewords of the dual of the $\mathrm{BCH}$ code that represent a basis of the code. The third step consist of adding redundant rows that resolve stopping sets corresponding to codewords of weight three and a subclass of codewords of weight four in the Hamming code. The fourth step introduces into the parity-check matrix a set of redundant rows obtained by a greedy search strategy, designed to completely resolve stopping sets of size four.

The generalized HT algorithm is summarized below.

Step 1) A redundant parity-check matrix for a Hamming code that resolves all stopping sets that correspond to correctable erasure patterns of size up to (but not including) $\sigma=4$ is created first. To this end, a generic $(\bar{m}, \bar{\sigma})$ erasure correcting set with $\bar{m}=\log _{2}(n+1)$ and $\bar{\sigma}=4$ is multiplied with the standard parity-check matrix of the Hamming code. The resulting matrix contains only unresolved stopping sets of size $\sigma=3$ and $\sigma=4$, which either correspond to a 
codeword of the Hamming code or contain the support of one such codeword in their support. We refer to this matrix as a generic erasure correcting matrix, and denote it by $\boldsymbol{H}_{\mathrm{h}, \mathrm{g}}$. Figure 7 visualizes the first step of the construction procedure.

Step 2) The matrix obtained in Step 1 is augmented to form a parity-check matrix of a BCH-code with minimum distance $d=5$. This is achieved by adding the binary expansion of the row vector

$$
\boldsymbol{h}_{\mathrm{BCH}}=\left(\begin{array}{llll}
\alpha^{0 \cdot(1+c)} & \alpha^{1 \cdot(1+c)} & \ldots & \alpha^{(n-1)(1+c)}
\end{array}\right),
$$

where $c$ is chosen according to the standard procedure for generating $\mathrm{BCH}$ codes (one common choice being $c=2$ ). Here, $\alpha$ is as defined in Section IV-C.2. We refer to this second component of the redundant parity-check matrix of the $\mathrm{BCH}$ code as $\boldsymbol{H}_{\mathrm{BCH}}$. Figure 8 visualizes the second step of the construction procedure.

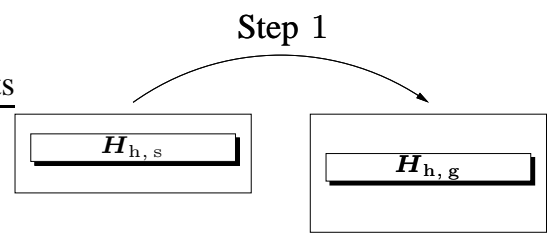

Fig. 7. Step 1: Transform $\boldsymbol{H}_{\mathrm{h}, \mathrm{s}}$ into a generic $(\bar{m}, \bar{\sigma})$ erasure correcting set $\boldsymbol{H}_{\mathrm{h}, \mathrm{g}}$.

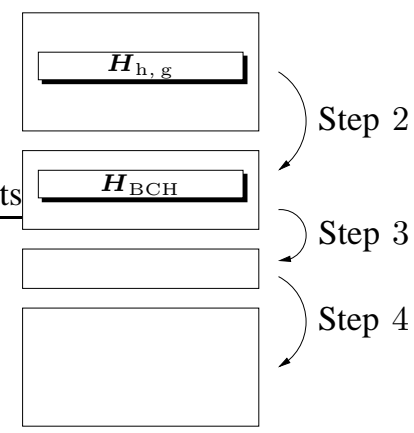

Fig. 8. Steps 2-4: Append $\boldsymbol{H}_{\mathrm{BCH}}$ (Step 2), and add code-defining parity-check equations to $\boldsymbol{H}_{\mathrm{h}, \mathrm{g}}$ (Steps 3 and 4).

Step 3) In this step, an additional set of redundant rows is added to the matrix in order to resolve all stopping sets of size $\sigma=3$ and a subset of those of weight $\sigma=4$. To this end, we use the fact that all unresolved stopping sets of size three and four correspond to codewords of the Hamming code.

Let $\boldsymbol{t}$ denote the support of an unresolved stopping set of size $\sigma=3$. The restriction of $\boldsymbol{H}_{\mathrm{h}, \mathrm{g}}$ to $\boldsymbol{t}$ equals

$$
\left[\left(\alpha^{a}\right)\left(\alpha^{b}\right)\left(\alpha^{a}+\alpha^{b}\right)\right]
$$

with $0 \leq a, b<n-2, a \neq b$, whereas the restriction of $\boldsymbol{H}_{\mathrm{BCH}}$ to $\boldsymbol{t}$ equals

$$
\left[\left(\alpha^{3 a}\right)\left(\alpha^{3 b}\right)\left(\left(\alpha^{a}+\alpha^{b}\right)^{3}\right)\right]
$$

where we have set $c=2$.

Consider first all stopping sets for which $\alpha^{3 a}=\alpha^{3 b}$.

Lemma V.1: In a finite field with characteristic two and order $n+1$, for any primitive element $\alpha, \alpha^{3 a}=\alpha^{3 b}$ implies $\left(\alpha^{a}+\alpha^{b}\right)^{3}=\alpha^{3 a}$.

Proof: Since the field has characteristic two, one can write

$$
\left(\alpha^{a}+\alpha^{b}\right)^{3}=\alpha^{3 a}+3 \alpha^{2 a+b}+3 \alpha^{a+2 b}+\alpha^{3 b}=\alpha^{2 a+b}+\alpha^{a+2 b} .
$$

Consequently,

$$
\alpha^{2 a+b}+\alpha^{a+2 b}=\alpha^{3 a}\left(\alpha^{b-a}+\alpha^{a-b}\right) .
$$

As $3 a-3 b=j n$, for some integer $j \geq 0$, it follows that $a-b=j n / 3$ and $b-a=2 j n / 3$. To complete the proof, we first prove the next more general claim: for each $u \in\{2 k+1 \mid k \in \mathbb{N}\}$, it holds that $\sum_{i=1}^{u-1} \alpha^{i n / u}=1$. The result of the lemma follows by specializing $u=3$. 
We prove the claimed result by showing that

$$
\left(\sum_{i=1}^{u-1} \alpha^{i n / u}\right)^{2}=\sum_{i=1}^{u-1} \alpha^{2 i n / u}=\sum_{i=1}^{u-1} \alpha^{i n / u} .
$$

To this end, observe that

$$
\begin{aligned}
\sum_{i=1}^{u-1} \alpha^{2 i n / u} & =\sum_{i=1}^{(u-1) / 2} \alpha^{2 i n / u}+\sum_{i=(u+1) / 2}^{u-1} \alpha^{2 i n / u} \\
& =\sum_{i=1}^{(u-1) / 2} \alpha^{2 i n / u}+\sum_{i^{\prime}=1}^{(u-1) / 2} \alpha^{2\left(i^{\prime}+u / 2-1 / 2\right) n / u} \\
& =\sum_{i=1}^{(u-1) / 2} \alpha^{2 i n / u}+\sum_{i^{\prime}=1}^{(u-1) / 2} \alpha^{\left(2 i^{\prime}-1\right) n / u} \\
& =\sum_{i=1}^{u-1} \alpha^{i n / u},
\end{aligned}
$$

where we have introduced $i^{\prime}=i-u / 2+1 / 2$ and used the fact that $\alpha^{n}=1$. This completes the proof.

Assume that $n / 3$ is an integer. Then there exist exactly $n / 3$ stopping sets corresponding to Hamming codewords of weight three for which $\alpha^{3 a}=\alpha^{3 b}$, as $a$ can only be chosen in the range $0<a<n / 3$. If we switch to the binary representation of the parity-check matrix, it is easy to see that the restriction of $\boldsymbol{H}_{\mathrm{h}, \mathrm{g}}$ to $\boldsymbol{t}$ only contains rows of weight zero or two, whereas the same restriction of $\boldsymbol{H}_{\mathrm{BCH}}$ only contains rows of weight zero or three. As a result, each stopping set corresponding to a weight-three codeword of the Hamming code for which $\alpha^{3 a}=\alpha^{3 b}$ can be resolved by an appropriate linear combination of one row from $\boldsymbol{H}_{\mathrm{h}, \mathrm{g}}$ and one row of $\boldsymbol{H}_{\mathrm{BCH}}$. We conduct the search for the appropriate rows in these matrices in a greedy fashion, i.e. by eliminating as many stopping sets as possible with each redundant row. Figure 8 visualizes this step.

Step 4) Step 3 gives rise to parity-check matrices that resolve all stopping sets of size $\sigma=3$, for which $\alpha^{3 a}=\alpha^{3 b}$ and $n / 3$ is an integer. As an example, this claim is true when $n=63$ and the corresponding code is a $[63,51,5] \mathrm{BCH}$ code. In order to resolve stopping sets that do not satisfy these constraints, we use greedy computer search techniques to identify a collection of redundant parity-checks suitable for accomplishing this goal. These checks are added to the concatenation of $\boldsymbol{H}_{\mathrm{h}, \mathrm{g}}, \boldsymbol{H}_{\mathrm{BCH}}$, and the parity-checks found in Step 3.

Acknowledgment: The authors are grateful to the anonymous reviewers for their constructive comments that significantly improved the exposition of the work. In addition, they would like to thank Dr. Richardson for handling the manuscript.

\section{REFERENCES}

[1] C. Di, D. Proietti, I. Telatar, T. Richardson, and R. Urbanke, "Finite-length analysis of low-density parity-check codes on the binary erasure channel," IEEE Trans. on Inform. Theory, vol. 48, no. 6, pp. 1570-1579, June 2002.

[2] M. Schwartz and A. Vardy, "On the stopping distance and stopping redundancy of codes," IEEE Trans. on Inform. Theory, vol. 52, no. 3, pp. 922- 932, March 2006.

[3] K. Abdel-Ghaffar and J. Weber, "Complete enumeration of stopping sets of full-rank parity-check matrices of Hamming codes," IEEE Trans. on Inform. Theory, vol. 53, no. 9, pp. 3196-3201, September 2007

[4] J. Han and P. Siegel, "Improved upper bounds on stopping redundancy," IEEE Trans. on Inform. Theory, vol. 53, no. 1, pp. 90-104, January 2007.

[5] T. Hehn, S. Laendner, O. Milenkovic, and J. B. Huber, "The stopping redundancy hierarchy of cyclic codes," in Proceedings of the 44th Annual Allerton Conference on Communication, Control and Computing, Allerton House, UIUC, Illinois, USA, September 2006, pp. 1271-1280.

[6] J. Han, P. Siegel, and A. Vardy, "Improved probabilistic bounds on stopping redundancy," submitted to IEEE Trans. on Inform. Theory, July 2007.

[7] O. Milenkovic, E. Soljanin, and P. Whiting, "Stopping and trapping sets in generalized covering arrays," in Proceedings of the 40th annual Conference on Information Sciences and Systems (CISS), Princeton University, Princeton, New Jersey, USA, March 2006, pp. $259-264$.

[8] T. Wadayama, "Average stopping set weight distribution of redundant random matrix ensembles," in Proceedings of Int. Symp. on Inform. Theory (ISIT), Nice, France, June 2007, pp. 2931-2935.

[9] J. Weber and K. Abdel-Ghaffar, "Stopping and dead-end set enumerators for binary Hamming codes," in Proceedings of the Twenty-sixth Symp. on Inform. Theory in the Benelux, Brussels, Belgium, May 2005, pp. 165-172.

[10] H. Hollmann and L. Tolhuizen, "On parity-check collections for iterative erasure decoding that correct all correctable erasure patterns of a given size," IEEE Trans. on Inform. Theory, vol. 53, no. 2, pp. 823-828, February 2007.

[11] F. MacWilliams and N. Sloane, The Theory of Error-Correcting Codes. North-Holland Publishing Company, 1977.

[12] K. M. Krishnan and P. Shankar, "On the complexity of finding stopping set size in Tanner graphs," in 40th Annual Conference on Information Sciences and Systems, March 2006, pp. 157-158.

[13] A. McGregor and O. Milenkovic, "On the hardness of approximating stopping and trapping sets in LDPC codes," in Proceedings of the IEEE Information Theory Workshop (ITW 2007), Lake Tahoe, September 2007.

[14] K. Dohmen, Improved Bonferroni Inequalities via Abstract Tubes. Springer-Verlag, 2003.

[15] S. Kwerel, "Most stringent bounds on aggregated probabilities of partially specified dependent probability systems," J. Amer. Statist. Assoc., vol. 70, pp. 472-479, 1975.

[16] N. Alon and J. Spencer, The Probabilistic Method, ser. Interscience Series in Discrete Mathematics and Optimization. John Wiley, 2000. 
[17] D. Deng, D. Stinson, and R. Wei, "The Lovász local lemma and its applications to some combinatorial arrays," Designs, Codes and Cryptography, vol. 32, no. 1-3, pp. 121-134, May 2004.

[18] D. B. Jaffe, "Information about binary linear codes," Web-database, http://www.math.unl.edu/ djaffe2/codes/webcodes/codeform.html.

[19] S. Lin and D. Costello, Error Control Coding, Second ed. Pearson Education, Inc., 2004.

[20] M. Tomlinson, C. Tjhai, M. Ambroze, and M. Ahmed, "Binary cyclic difference set codes derived from idempotents based on cyclotomic cosets," submitted to IEEE Trans. on Inform. Theory, May 2004.

[21] M. Hall, Jr., Combinatorial Theory. Wiley, 1998.

[22] N. Santhi and A. Vardy, "On the effect of parity-check weights in iterative decoding," in Proc. of the IEEE Internat. Symp. on Inform. Theory, Chicago, Illinois, July 2004, p. 322.

[23] T. Halford and K. Chugg, "Random redundant soft-in soft-out decoding of linear block codes," in Proceedings of Int. Symp. on Inform. Theory (ISIT), Seattle, WA, July 2006, pp. 2230-2234.

[24] J. Key, "Some recent developments in permutation decoding," [Online]. Available at http://www.ces.clemson.edu/ keyj/Key/Cork06.pdf, 2006.

[25] J. Wolfmann, "A permutation decoding of the $(24,12,8)$ golay code," IEEE Trans. on Inform. Theory, vol. 29, no. 5, pp. 748-750, September 1983.

[26] J. Schoenheim, "On coverings," Pacific J. Math, vol. 14, no. 4, pp. 1405-1411, 1964.

[27] H. Pishro-Nik and F. Fekri, "On decoding of low-density parity-check codes over the binary erasure channel," IEEE Trans. on Inform. Theory, vol. 50, no. 3, pp. 439-454, March 2004

[28] O. Milenkovic, "Private communication with J. Han and P. Siegel," 2006.

[29] A. Hedayat, N. Sloane, and J. Stufken, Orthogonal Arrays: Theory and Applications. New York: Springer Verlag, 1999.

[30] V. Tarakanov, "Difference sets," Encyclopedia of Mathematics, Springer Link, available online at http://eom.springer.de/d /d031760.htm\#d031760_00c1. 